\title{
1 Liquid-liquid phase separation of tau driven by hydrophobic interaction facilitates fibrillization of tau
}

4 Yanxian Lin1, ${ }^{*}$, Yann Fichou2, ${ }^{*}$, Andrew P. Longhini ${ }^{3}$, Luana C. Llanes2,4, Yinson Yin2, Guillermo

5 C. Bazan5, Kenneth S. Kosik ${ }^{3,6}$, Songi $\operatorname{Han}^{2,7} \dagger$

6

7 1 Biomolecular Science and Engineering, University of California, Santa Barbara, CA 93106

82 Department of Chemistry and Biochemistry, University of California, Santa Barbara, CA 93106

93 Molecular, Cell and Developmental Biology, University of California, Santa Barbara, CA 93106

$10{ }_{4}$ Center for Polymers and Organic Solids, University of California, Santa Barbara, CA 93106

115 Departments of Chemistry and Chemical Engineering, National University of Singapore, 117543

12 Singapore

13 6 Neuroscience Research Institute, University of California, Santa Barbara, CA 93106

147 Department of Chemical Engineering, University of California, Santa Barbara, CA 93106

15

$16 *$ equal contributions.

$17 \uparrow$ corresponding author: Songi Han. Email: songihan@ucsb.edu 


\section{Abstract}

21 Amyloid aggregation of tau protein is implicated in neurodegenerative diseases, yet its facilitating

22 factors are poorly understood. Recently, tau has been shown to undergo liquid liquid phase

23 separation (LLPS) both in vivo and in vitro. LLPS was shown to facilitate tau amyloid aggregation

24 in certain cases, while independent of aggregation in other cases. It is therefore important to

25 understand the differentiating properties that resolve this apparent conflict. We report on a model

26 system of hydrophobically driven LLPS induced by high salt concentration (LLPS-HS), and

27 compare it to electrostatically driven LLPS represented by tau-RNA/heparin complex coacervation

28 (LLPS-ED). We show that LLPS-HS promotes tau protein dehydration, undergoes maturation and

29 directly leads to canonical tau fibrils, while LLPS-ED is reversible, remains hydrated and does not

30 promote amyloid aggregation. We show that the nature of the interaction driving tau condensation

31 is the differentiating factor between aggregation-prone and aggregation-independent LLPS.

\section{Introduction}

34 Tau is a protein mainly present in the central nervous system that binds and stabilizes microtubules

35 in neurons [1]-[3]. Under healthy conditions, tau is soluble across a wide range of temperatures, 36 protein concentrations, $\mathrm{pH}$ and ionic strengths of the buffer, while under pathologic conditions, 37 tau undergoes amyloid aggregation that yields insoluble, irreversible and cross- $\beta$ rich fibrils. While 38 the functions of tau are not fully understood, in the healthy state tau is tightly bound to 39 microtubules [4] and the concentration of the unbound tau protein is exceptionally low-on the 40 order of $1 \sim 10 \mathrm{nM}^{1}$. Under pathological conditions, intracellular tau transforms from its 41 microtubule-bound or soluble state to a fibrillar state. In fact, intracellular deposits of aggregated

\footnotetext{
${ }^{1}$ Estimated from tubulin concentration of $40 \mu \mathrm{M}$ [5], total tau concentration (bound and free) of $1 \sim 2 \mu \mathrm{M}$ [6], dissociation constant of $0.1 \sim 1 \mu \mathrm{M}$ [4] as well as stoichiometry of $\sim 0.5$ tau/tubulin dimer [7]. See Materials and Methods for calculation.
} 
42 tau are a diagnostic hallmark of a wide range of neurodegenerative diseases known as tauopathies

43 [8]. The mechanism by which low concentrations of tau is recruited to assemble into tangles

44 remains undetermined. Hence, factors that drive assembly and condensation, or generally

45 mechanisms that increase the intracellular local concentration of tau are of great interest to 46 understand this important pathological process.

47 Liquid-liquid phase separation (LLPS) is a process that is readily driven by multivalent weak interactions between flexible polymers, yielding a polymer-rich phase separated from a 49 polymer-depleted phase [9], [10], the former of which is usually observed as micrometer size 50 fluidic droplets. Here, we use the widest definition of polymers that hence includes intrinsically 51 disordered proteins (IDPs) and ribonucleic acid (RNA). LLPS has been used to describe the 52 formation of membraneless organelles in vivo that are proposed to play important roles in 53 mediating cellular functions [11], [12]. The interest in biomolecular condensates in the cellular 54 context has exploded in recent years, fueled by intriguing observations of LLPS of IDPs associated 55 with neurodegenerative diseases, including FUS, TDP-43, hnRNPA1, synuclein [13]-[18] as well as more recently tau [19]-[25]. Despite the ubiquitous occurrence of LLPS with IDPs, the physical

57 principles that govern their formation, phase diagrams or properties are not universal. LLPS of 58 IDPs can be driven by a variety of interaction types and with a wide range of interaction strengths.

59 The driving interaction for association that forms the basis for LLPS typically includes electrostatic charge-charge and dipole-dipole interactions between the backbone, charged/polar side chains and

61 polar solvent molecules, and hydrophobic interactions. The latter involve water-repelling protein 62 or polymer side chains that can be aromatic [26], non-polar [9], [27] or even charged residues [28], 63 [29] that, in the context of the protein or polymer surface, may display less favorable protein-water 64 interactions compared to protein-protein and water-water interactions. Molecular crowding, often 65 modeled by adding polyethylene glycol (PEG), can therefore enhance hydrophobic interactions by 66 removing hydration water from the dense LLPS phase [30]. However, the potentially differential 67 role of electrostatic and hydrophobic interactions for the LLPS of tau have neither been 68 investigated nor dissected to date. Recent work [9], [26], [31]-[35] has highlighted certain 69 interactions as particularly important in driving protein LLPS, in particular $\pi$ - $\pi$ and $\pi$-cation 70 interaction between charged and aromatic residues [36], [37]. Tau has few aromatic amino acids 71 ( $2 \%$ for full length tau $2 \mathrm{~N} 4 \mathrm{R})$ or arginine residues ( $\sim 3 \%$ for $2 \mathrm{~N} 4 \mathrm{R})$, consequently little possibility 72 to engage in $\pi-\pi$ or $\pi$-cation interactions. Nevertheless, tau's sequence exhibits a rich landscape of 
73 chemical properties with polar, charged and hydrophobic residues present throughout the protein.

74 There is a need to study the driving forces of tau LLPS and uncover what role they play in 75 pathology.

LLPS has been shown to promote amyloid aggregation of FUS and hnRNPA1 [13]-[16]. The verdict is not so clear for LLPS of tau. Ambadipudi et al. in 2017 [19] and Wegmann et al. in 2018 [20] proposed that tau in a LLPS state actively promoted amyloid aggregation, while a recent study from our group [38] showed that tau in LLPS formed by complex coacervation with RNA does not induce or protect from aggregation. Herein, we extensively explore the hypothesis that the nature of the interactions driving tau LLPS is the main factor that influence how LLPS impact amyloid aggregation.

We and others have previously shown that tau undergoes electrostatically driven LLPS (LLPS-ED), either with itself — in a process known as simple coacervation (SC) —or with polyanions - in a process known as complex coacervation (CC) [21], [22], [33], [38]. Meanwhile, tau can undergo LLPS upon addition of high concentration of salt (LLPS-HS)[38]. Concentrated salt (e.g. of $\mathrm{NH}_{4+}, \mathrm{K}+$ or $\mathrm{Na}+$ along the Hofmeister series) has been shown to exert a "salting-out" effect [39], [40], presumably by dehydrating the protein and promoting entropy-driven hydrophobic interactions among proteins [41], [42]. High concentration of salt including $\mathrm{NaCl}$ has been found to induce LLPS of several proteins, including BSA [43], lysozyme [44] and most

92 induced by adding high concentration of salt (LLPS-HS) as a model for tau LLPS driven by 93 hydrophobic interactions. Using EPR spectroscopy, fluorescence spectroscopy and biochemical 94 assays, we compared the properties of tau in LLPS-HS with that of LLPS-ED formed by tau-tau 95 or tau-RNA to uncover whether there is a cause-effect relationship between the LLPS and 96 aggregation of tau.

\section{RESULTS}

\section{Tau undergoes LLPS at high salt concentration} conditions, displaying both high solubility and stability. The charge and Hopp-Woods 
hydrophobicity plots (both are moving-averaged over 25 consecutive residues) show that $2 \mathrm{~N} 4 \mathrm{R}$ consists of a hydrophilic and negatively charged N-terminal half and a weakly hydrophobic and overall positively charged C-terminal half (Figure 1A). The four repeat domains (R1-R4) are responsible for binding microtubules and constitute a large part of the core of amyloid fibrils found in vivo and in vitro [46]. The goal of our study is to investigate tau LLPS based on hydrophobic interactions separately from those based on electrostatic interactions. To maximize the hydrophobicity while keeping the repeat domains, we studied the LLPS of N-terminal-truncated 2N4R between residues 255-441, referred to as tau187 (Figure 1A). To mute potential effects of covalent disulfide bonding, we introduced double site mutations to the two native cysteines, C291S/C322S. Unless stated explicitly, we refer to tau187C291SC322S as tau187, and to 2N4RC291SC322S as 2N4R throughout this work.

To eliminate electrostatic interactions, we mixed tau with excess amount of $\mathrm{NaCl}$. Both tau187 and 2N4R samples became turbid and formed abundant droplets when $[\mathrm{NaCl}]$ was adjusted to above $4 \mathrm{M}$ and $4.75 \mathrm{M}$, respectively (Figure 1B). After formation, droplets merged within seconds, confirming their fluidity (Figure 1C). We refer to this process of high salt concentrationinduced LLPS as LLPS-HS, and the resulting droplets as high salt droplets in this article. We furthermore confirmed that both tau187C291S and tau187C291SP301L form LLPS-HS (Figure 1figure supplement 1), showing that inter-tau disulfide bonding and aggregation-prone mutations do not prevent LLPS-HS.

We have previously shown that tau187 undergoes LLPS-ED, and established a phase diagram using a combination of experiments, theory underlying coacervation and field theoretic simulations (FTS) of LLPS [22]. The LLPS-ED phase (formed either by SC or CC) typically dissolves at a total salt concentration above [ $\mathrm{NaCl}] \sim 100-200 \mathrm{mM}$ [22], [33], while the LLPS-HS obviously only forms above a much higher salt concentration (3-4 M; Figure 1B). The question we ask is whether the properties of the protein tau and the LLPS phase are different between the two forms of LLPS. We start by testing the sensitivity of the dense liquid phase to 1,6-hexanediol 129 (1,6-HD), an amphiphilic small molecule that is known to disrupt weak hydrophobic interactions, [47]-[49] which has been widely used to verify the formation and dissolution of various forms of LLPS, including that of FUS [50] and of tau [20], [51]. We prepared tau LLPS-ED in two ways: by mixing tau187 with RNA by the CC mechanism, or by lowering the ionic strength of the 
When adding 4\% 1,6-HD, neither form of tau LLPS-ED, tau-RNA droplets formed by CC or tau low-salt droplets formed by SC, dissolved. In contrast, tau LLPS-HS dissolved completely upon addition of 4\% 1,6-HD (Figure 1D).

The distinct effect of 1,6-HD demonstrates that the tau molecules are held together in the condensed phase of LLPS by different types of interactions in LLPS-ED versus in LLPS-HS. The observation that the stability of LLPS-ED, whether formed of tau187-RNA CC or of 2N4R SC, is insensitive to 1,6-HD confirmed that the interactions holding tau together in the condensed phase is not weakened by an amphiphilic molecule; this behavior is expected for purely electrostatic associations. Considering that the Debye length at $4 \mathrm{M} \mathrm{NaCl}$ is $\sim 0.1 \mathrm{~nm}$, which is much smaller than the diameter of a water molecule [52], [53], electrostatic interactions between tau molecules are muted in LLPS-HS. Besides electrostatic screening, salts made of $\mathrm{Na}+$ and $\mathrm{Cl}$ - at these concentrations also exert dehydration induced salting out of proteins following the Hofmeister series. Together with the observation that 1,6-HD dissolves high salt droplets, these results demonstrate that LLPS-HS are predominantly hydrophobically driven. Hydrophobic interactions arise from weaker protein-water compared to water-water and protein-protein interactions, resulting in (i) water to be readily expelled from the protein surface to rather hydrogen bond with other solvent molecules, i.e. other water molecules in the bulk, and (ii) protein molecules to interact via van der Waals interactions. Amphiphilic molecules such as 1,6-HD can restore interactions between protein and water and/or disrupt interaction between associated proteins, disrupting the hydrophobic driving forces for LLPS-HS. These results verify that LLPS-HS is a good model system to study the consequences of hydrophobically driven LLPS on the irreversible process of aggregation.

Many examples of protein LLPS have been shown to undergo irreversible maturation [16], [54]. To study the reversibility property of LLPS-HS, we recorded turbidity of high salt droplet samples upon repeated heating-cooling cycles. At $3.5 \mathrm{M} \mathrm{NaCl}$, turbidity at $\mathrm{T}<35^{\circ} \mathrm{C}$ remains near zero and significantly increases to 0.4 at $\mathrm{T}>40{ }^{\circ} \mathrm{C}$, indicating a lower critical saturation temperature behavior (LCST) where LLPS is thermodynamically more favorable at higher temperature (Figure 2-figure supplement 1A). When cooled down to low temperature, the original transparent sample remained slightly turbid (Figure 2-figure supplement 1A), a second heating-cooling cycle 
showed further increase of remaining turbidity. In contrast, tau-RNA LLPS-ED formed by CC showed no remaining turbidity after consecutive heating-cooling cycles (Figure 2-figure supplement 1B). The same results were found with 2N4R (Figure 2-figure supplement 1C). The hysteresis in the build-up of turbidity with repeated heating-cooling cycle implies that LLPS-HS leads to partially irreversible associations between tau, while LLPS-ED formation and melting is reversible.

To further probe the micro-environment inside droplets, we labeled tau with an in-house synthesized fluorescence molecular rotor derived from a boron-dipyrromethene (BODIPY) core [55] and performed Fluorescence Lifetime Image Measurements (FLIM) over the course of 48 hours (See Materials and Methods for synthesis and microscopy details). Molecular rotors are a class of dyes whose fluorescent lifetimes are sensitive to the local microviscosity of their environment, with a low lifetime indicating a fluid, water-like environment and a high lifetime representing a viscous environment [56]-[58]. Further, within a limited regime, the relationship between the fluorescent lifetime and the microviscosity follows the Förster-Hoffman equation (see calibration between fluorescent lifetime and viscosity in Figure 2-figure supplement 2). Unlike viscosity that describes the bulk property of a liquid, microviscosity describes the friction that, in this case, is experienced by a single biomolecule due to its local environment. The molecular rotor was conjugated to 2N4R, as described in Materials and Methods. FLIM images were collected on a confocal microscope and a pixelwise fit of the images binned into histograms. Shortly after triggering LLPS, 2N4R LLPS-HS droplets showed a Gaussian distribution of lifetimes centered around 4.08 $\pm 0.32 \mathrm{~ns}(916 \mathrm{cP})$. In comparison, 2N4R LLPS-ED droplets formed by CC between 2N4R and RNA (Figure 2E, F) showed a lifetime centered around 3.55 $\pm 0.07 \mathrm{~ns}$ (195 cP). In other words, 2N4R in LLPS-HS experiences a higher microviscosity than in LLPS-ED. After a 48 hour incubation period, the average fluorescence lifetime of 2N4R in LLPS-HS samples increased from $4.08 \pm 0.26$ to $4.15 \pm 0.13 \mathrm{~ns}$ (Figure $2 \mathrm{~F}$ ), while that of $2 \mathrm{~N} 4 \mathrm{R}$ in LLPS-ED decreased from $3.55 \pm 0.07$ ns to $3.39 \pm 0.12$ ns (Figure 2E, F). Concurrently, amorphous, non-spherical condensates formed within LLPS-HS, while the overall droplet shape of LLPS-ED (of 2N4R-RNA CC) remains spherical (Figure 2B, D). The emergence of non-spherical structures within the droplets and the increase in microviscosity sensed by the tau protein indicate that LLPS-HS undergo maturation of

195 the condensate. LLPS-ED on the other hand retains liquid properties that appears to slightly less viscous over time. 


\section{LLPS-HS triggers canonical tau amyloid aggregation}

To test the hypothesis that maturation of droplets is associated with amyloid formation in the sample, we incubated high salt droplets with a trace amount of Thioflavin T (ThT). While high salt droplets emerge spontaneously and fully upon preparing samples under droplet-forming conditions, ThT fluorescence starts from baseline and steadily increases over $\sim 24$ hour duration (Figure 3A). The increase of ThT fluorescence reflects an increase in cross- $\beta$ sheet content developing over hours. Subsequent imaging of tau samples-in LLPS-HS conditions after overnight incubation - showed fibril-like species by TEM that are visually similar to tau-heparin fibrils (Figure 3B). This supported the hypothesis that LLPS-HS is followed by the amyloid aggregation of tau.

To show the connection between LLPS-HS and amyloid aggregation, we incubated tau at equal concentration, while varying $[\mathrm{NaCl}]$ on a multi-well plate. We recorded bright field microscope images before incubation showing the amount of high salt droplets, after which we monitored ThT fluorescence over time (Figure 3A). The abundance of droplets visually observed via bright field microscope imaging immediately after sample preparation (at $t=0)$ strongly correlates with the ThT fluorescence intensity after incubation ( $t=24$ hours). The same results were found with 2N4R (Figure 3-figure supplement 2). The initial turbidity (at $500 \mathrm{~nm}$ ) that quantifies the volume fraction of high salt droplets at $t=0$ (Figure SI 10 in [38]) was plotted against final ThT fluorescence readings at $t=24$ hours, representing the relative amount of amyloid aggregates formed (Figure 3C). We found a proportional correlation (linear $\mathrm{R}_{2}$ of 0.43 ) between the initial turbidity and the final ThT fluorescence readings (Figure 3C). This result implies that LLPS-HS causally triggers amyloid formation. In contrast, electrostatically driven LLPS neither promotes RNA-assisted tau aggregation, nor prevents heparin-induced tau aggregation as shown in Figure 3C and Figure 3-figure supplement 1, and as previously reported [38]. These results show that the strong correlation between LLPS and amyloid aggregation is unique to

224 hydrophobically-driven LLPS, as represented by LLPS-HS, while LLPS-ED and aggregation are independent processes.

We further studied the effects of the frontotemporal dementia-related mutation P301L of tau on the correlation between LLPS-HS and aggregation. Our data showed that tau187P301L 
quantity at $t=0$ (Figure 3C), implying that for a similar initial volume of droplets, tau187P301L generates higher quantities of cross $\beta$-sheet structures, resulting in higher ThT fluorescence intensity. Meanwhile, an analysis of the aggregation half time showed that in all examined conditions P301L has significantly shortened the half time (Figure 6-figure supplement 2). These results showed that P301L promotes tau aggregation under LLPS-HS conditions, consistent with its broadly known aggregation-promoting properties under various in vitro and in vivo conditions. Similar results were observed with 2N4R (Figure 6-figure supplement 1).

\section{Dehydration facilitates hydrophobically driven LLPS-HS}

To reveal the mechanisms that promote the transition of tau from high salt droplet to tau in fibrils, we next focused on investigating the driving forces for tau to form in LLPS-HS. The dissolution by 1,6-HD showed high salt droplets to be held together by hydrophobic interaction (Figure 1D). When increasing the amount of 1,6-HD, we found the minimum $[\mathrm{NaCl}]$ to induce LLPS-HS to shift significantly towards higher $\mathrm{NaCl}$ concentration (Figure 4-figure supplement 3C). This implies that a greater amount of salt is needed to restore the same enhancement of hydrophobic interactions that are disrupted by 1,6-HD.

Turbidity shows LLPS-HS is favored at higher temperature, following lower critical saturation temperature behavior (LCST) (Figure 2-figure supplement 1A, C). This suggests that free energy for LLPS-HS is rendered negative $(\Delta G<0)$ by a dominant and positive entropy term, $\mathrm{T} \Delta \mathrm{S}$, according to $\Delta \mathrm{G}=\Delta \mathrm{H}-\mathrm{T} \Delta \mathrm{S}$. LCST behavior is seen when phase separation is accompanied by an increase in total entropy (i.e. $\Delta S>0$ ) that, as a result, leads to an increasingly dominant and positive entropy $(\mathrm{T} \Delta \mathrm{S})$ term at higher temperature. The temperature at which $\Delta \mathrm{G}=0$ is then the phase transition temperature, cloud point $\mathrm{T}_{\mathrm{cp}}$, above which phase separation occurs spontaneously.

254 Interestingly, either increasing $[\mathrm{NaCl}]$ or increasing [tau] lowers the $\mathrm{T}_{\mathrm{cp}}$ above which LLPS-HS occurs (Figure 4-figure supplement 1). This finding suggests that both the addition of salt and higher tau concentration contribute to entropy gain upon LLPS. When adding PEG as a molecular

257 crowder, we observed that the minimum $[\mathrm{NaCl}]$ necessary to induce LLPS-HS also shifted towards lower concentrations (Figure 4-figure supplement 3B). A recent study by Park et al. 
LLPS without partitioning into the dense phase of LLPS [30]. Taken together, our results are consistent with $\mathrm{NaCl}$ and $\mathrm{PEG}$ both sequestering water, and hence depleting the protein system of free water in a process known as salting out [41], [59]. Dehydration of the tau-water system will facilitate hydrophobic interactions and subsequent condensation by an increase of the entropic term $\mathrm{T} \Delta \mathrm{S}$.

While the observations made by systematic changes in $[\mathrm{NaCl}],[\mathrm{tau}],[\mathrm{PEG}]$ and temperature is consistent with dehydration- and entropy-driven LLPS-HS, a more direct observation of dehydration is more satisfying. To measure the effect of dehydration near the tau protein surface, we labeled tau with unpaired electron spin labels to perform electron spin envelope echo modulation (ESEEM) and Overhauser dynamic nuclear polarization (ODNP) measurements. ESEEM measures the relative changes in the local water density within a $\sim 3-6 \AA$ shell around a spin label [60], [61], while ODNP measures water accessibility [62] and translational diffusivity within a shell of $\sim 5-10 \AA$ [63], [64] (Figure 4A).

We labeled tau187 with MTSL on site 313 within the R3 domain and dissolved it into a deuterium-based buffer. The magnitude of the ESEEM deuterium peak intensity dropped by over $20 \%$, immediately upon addition of $\mathrm{NaCl}$ that led to the formation of high salt droplets (Figure 4B). This result represents a significant reduction of local water density near site 313.

We furthermore carried out ODNP relaxometry measurements to determine the crossrelaxivity parameter $\mathrm{k}_{\sigma}$, for tau187 at sites 303, 313, 322 and 404, representing the $4 \mathrm{R}$ domain and the $\mathrm{C}$-terminal region of tau. The $\mathrm{k} \sigma$ value reflects on local water accessibility and/or translational diffusivity within $\sim 1 \mathrm{~nm}$ of the spin label (Eq. 5 of [65]). We found that the value of ko dropped from 31 - 56 M-1S-1 to 9 - 23 M-1S-1 for all four sites as soon as high salt droplets formed (see per site trend in Figure 4C). Note that $\mathrm{k}_{\sigma}$ measured on the free radical 4-hydroxyl-TEMPO was unchanged upon addition of salt at concentrations that induce LLPS-HS (Figure 4C). Therefore,

287 the decrease of $\mathrm{k} \sigma$ observed reflects a change in hydration properties near the tau protein surface upon LLPS-HS formation. The reduction of the deuterium peak magnitude in ESEEM at site 313 shows that the reduction of water accessibility contributes to the drop of k $\sigma$. Given the $\sim 25 \%$ 
source data 1), it is reasonable to assume that it reflects, in addition to local dehydration, a significant slowdown of surface water diffusivity near the microtubule binding domain and Cterminal of tau when condensed into LLPS-HS. Strikingly, k $\sigma$ remains unchanged upon formation of tau-RNA LLPS-ED (Figure 4C), showing that dehydration of LLPS-internal water is a characteristic associated with hydrophobically driven LLPS-HS, but not of LLPS-ED.

\section{Dehydration reduces tau dynamics and triggers oligomer formation}

To study the dynamics of tau within high salt droplets, we carried out continuous wave (cw) EPR spectroscopy. We labeled tau187 at four different sites: 303, 313, 322 and 404, and extracted the rotational correlation times of the tethered spin label, tr, from fitting the cwEPR lineshape (see Materials and Methods). The value for $t R$ of the tethered spin reflects on the local protein mobility, as the spin label is tethered to the protein side chain with steric constraints [66]. We first looked at tr of the four sites before and after forming LLPS-HS. We found that LLPS-HS formation results in dramatic changes in the spectral lineshape of the spin labeled tau (Figure 5A,

Figure 5-figure supplement 2). We used the microscopic order macroscopic disorder (MOMD) model [67] with incremental complexity to fit the cw EPR lineshapes, using the MultiComponent program developed by Christian Altenbach. At $[\mathrm{NaCl}]=0-1.5 \mathrm{M}$ (no droplets), lineshapes of the samples were well fit with one isotropic tR component (Figure 5-figure supplement 2). Increasing $[\mathrm{NaCl}]$ to $3 \mathrm{M}$ and above resulted in LLPS-HS. The lineshape of the samples deviated from the one component fit, and required an additional mobility component (Figure 5-figure supplement 1A). The extracted tR and the population of each component is represented in Figure 5B as a function of salt concentration. We found that tR of the second component was significantly higher than for the first component (i.e. slower dynamics), for all sites. The second component represented $65-96 \%$ of the spin label population above $3 \mathrm{M} \mathrm{NaCl}$ (Figure 5-source data 1).

316 Meanwhile, increasing $[\mathrm{NaCl}]$ up to $4.5 \mathrm{M}$ alone does not change the lineshape of the free radical 317 4-hydroxyl-TEMPO (Figure 5-figure supplement 4), showing the effects of high salt 318 concentration do not change the rotational dynamics of the spin itself. We hypnotize that this 319 increase of rotational correlation time tR reflect on the formation of higher molecular weight assemblies, i.e. oligomers. These results imply that the dynamics of the majority of tau proteins,

321 reported on via protein-tethered spin labels, is slowed down in the LLPS-HS state. In addition to 322 the slowed rotational dynamics, we found emergence of rotational anisotropy in the second 
component for $[\mathrm{NaCl}]$ above $4 \mathrm{M}$. The presence of anisotropy indicates that the tethered spin label is highly restricted [67], [68], likely from steric hindrance of nearby tau protein, suggesting that tau starts to more tightly pack into protein assembly at very high salt concentration.

Next, we endeavor to show whether the formation of these oligomer species, revealed by slow label dynamics, originates from high salt concentration or from LLPS itself. First, in the absence of high salt droplets, we observed that the lineshape of cw EPR spectra of the tau-tethered spin label broadened upon $\mathrm{NaCl}$ addition, giving an average tR of $\sim 450$ ps without $\mathrm{NaCl}$ and $\sim 600$ ps at $1.5 \mathrm{M}$, at all sites (Figure 5-source data 1). In addition, at constant [ $\mathrm{NaCl}]$ we titrated [HD] from $0 \%$ to $10 \%$, where [HD] above $2 \%$ eliminated droplets. CwEPR lineshape analysis showed that the correlation time was neither abruptly nor distinctly reduced in the absence of droplets above 2\% HD (Figure 5-figure supplement 3). These results show that the drastic reduction in protein dynamics, which we hypothesized to reflect on the formation of oligomers, originate from dehydration by concentrated salt, only indirectly by LLPS.

\section{P301L promotes hydrophobic interactions}

One of the mysteries in tau amyloid aggregation is how a single site mutation, e.g, P301L, dramatically promotes the aggregation propensity of a protein of hundreds of amino acids. We propose here a plausible mechanism. We utilize LLPS-HS as a tool to measure hydrophobic interactions among tau. We studied the effects of the P301L mutation on LLPS-HS propensity. We measured turbidity of tau187 with and without P301L mutation under the exact same conditions. Turbidity from independent biological repeats showed that in the presence of an intermediate salt concentration of $3.25 \mathrm{M}$, the presence of P301L mutation significantly promoted LLPS-HS formation (Figure 6B). In other words, the P301L mutation lowers the $[\mathrm{NaCl}]$ threshold required to induce LLPS-HS. Representative microscope images, shown in Figure 6A, confirmed that P301L promotes high salt droplet formation. These results suggest that the P301L mutation promotes hydrophobic interactions among tau proteins, in agreement with previous proposal that P301L mutation extends local conformation, hence reveals the hydrophobic hexapeptide 306VQIVYK311 [69]. However, whether the increased hydrophobicity of tau in the presence of $\mathrm{P} 301 \mathrm{~L}$ is solely due to conformational changes or other biophysical factors requires in-depth 
studies in the future. In addition, we observed that the ThT activity triggered by LLPS-HS (shown

355 in Figure 3 for wild type (WT)) increases further for P301L compared to WT (Figure 6C). This enhanced ThT fluorescence for P301L mutant is most likely a combination of both the promotion of droplet formation (which in turn triggers ThT-sensitive aggregation) and a high aggregation propensity that facilitate the droplet to fibril transition.

\section{Discussion}

360 Protein liquid-liquid phase separation in pathological contexts have led to intense investigation in 361 the recent past. In particular, tau LLPS has been studied in several reports leading to contrasting 362 hypothesis whether or not LLPS directly triggers amyloid aggregation. We address in this study 363 two key questions: (1) Is tau LLPS of a universal type or could droplets formed in different 364 conditions possess different properties? (2) Is there a direct cause-and-effect relationship between LLPS and aggregation?

In this report, we have shown that tau at high salt concentration is capable of forming LLPS (referred to as LLPS-HS) driven by hydrophobic interactions. High $\mathrm{NaCl}$ concentrations both screen electrostatic interactions and sequester water away from the protein, thereby amplify the effect of hydrophobic association of tau. The concentration of salt needed to achieve LLPS of tau of the order of $\sim 3 \mathrm{M}$ is not physiological by any means. However, the influence of such condition

372 on the proteins or biopolymers, i.e. strong electrostatic screening and amplified hydrophobic 373 attraction can easily represent physiologically relevant state. For example, tau under conditions of 374 significant phosphorylation and acetylation would experience effects similar to that of screening 375 the net positive charge effects of the many lysine residues lining on the tau surface, while strong hydrophobic attraction was proposed to be at the origin of hyperphosphorylated tau aggregation $377[70]$.

Using EPR spectroscopy, FILM and biochemical assays, we showed that LLPS-HS exhibit slowed local dynamics, restricted water accessibility, elevated microviscosity, as well as irreversible maturation, in stark contrast to electrostatically driven LLPS-ED. Many different conditions can facilitate tau LLPS, but this study shows that the classification of hydrophobically or 
their properties or effects on aggregation. We used tau-RNA complex coacervation to showcase LLPS-ED, but as shown in Figure 1D and elsewhere [33], [34], both complex and simple coacervation can form LLPS-ED.

Establishing a causal relationship between LLPS and protein aggregation is challenging because the two processes can occur under identical conditions without necessarily being dependent [38]. Importantly, under conditions where both phenomena occur, it is tempting to deduce a cause-andeffect relationship. Firstly, droplets can form and dissolve within seconds to minutes, while aggregation occurs on the timescale of hours to days, therefore the sequential occurrence of droplets then aggregates does not necessarily imply LLPS is an intermediate state towards aggregation. Secondly, droplets, by definition, increase protein concentration. Therefore, even if both phenomena were completely independent, one would observe aggregates slowly forming in the inside of pre-existing droplets, merely because it is where the protein is, which might lead to the wrong conclusion that droplets directly trigger aggregation. In our opinion, a robust way to establish causation between droplets and aggregates is to examine multiple conditions across the LLPS phase boundary, and correlate the changes of droplet volume to the change of fibril mass. Using this approach, we have shown in [38] that although tau-cofactor LLPS-ED and amyloid aggregation are concomitant in many conditions, they are biophysically independent processes. In this study, we show that hydrophobically driven LLPS-HS is different, and that LLPS-HS of tau trigger amyloid formation (Figure 3C and Figure 3-figure supplement 3).

Previous reports on tau LLPS made with K18 at low salt concentrations showed that the droplets were dissolved above $200 \mathrm{mM} \mathrm{NaCl}$ and at $3 \% \mathrm{HD}$, suggesting that a combination of 407 electrostatic and hydrophobic driving forces were holding the liquid condensed state together [19], 408 [51]. These droplets were not reported to evolve spontaneously to ThT-active species. In contrast, 409 droplets formed by hyperphosphorylated 2N4R in Wegmann et al. [20] were shown to evolve to 410 amyloid fibrils and to be insensitive to $[\mathrm{NaCl}]>1 \mathrm{M}$, but to be sensitive to $10 \% \mathrm{HD}$, showing that 411 they are driven mostly by hydrophobic interactions. These results together with our work suggest 412 a general mechanism that hydrophobically-driven droplets promote aggregation. 
414 We have shown that the local environment in the high salt droplets is drastically different from the 415 one in tau LLPS-ED droplets. FLIM showed that, initially after high salt droplet formation, i.e. 416 even in the absence of significant aging, the micro-viscosity is significantly higher in LLPS-HS 417 than in LLPS-ED droplets. Furthermore, ESEEM and ODNP measurements revealed that water 418 accessibility and water dynamics is significantly more retarded in LLPS-HS than in LLPS-ED. 419 These properties found in LLPS-HS are favorable to protein aggregation because amyloid 420 formation requires a critical step of dehydration, where even hydrophilic residue must give up their 421 ideal hydration to pack into cross- $\beta$ sheets and steric zippers. Besides, amyloid fibers form a solid422 like matrix of high viscosity. We suggest that the features of high viscosity, perturbed protein 423 dynamics and dehydration are general hallmarks of protein LLPS on-pathway to aggregation.

$425 \mathrm{Cw}$ EPR linseshape analysis provides a mechanistic view of tau aggregation through LLPS-HS.

426 We found that increasing the concentration of salt progressively triggers the formation of high 427 order oligomers, as evidenced by a long rotational correlation time of the spin labels tethered to 428 tau. These oligomers are observed even at medium $[\mathrm{NaCl}]$ (e.g. $1.5 \mathrm{M}$ ) where droplets are not 429 present. An analysis of the dipolar broadening of the cwEPR spectra (Figure 5-figure supplement 430 4) shows the absence of specific packing (such as in-register cross- $\beta$ sheets or steric zipper 431 structures) in these ThT-inactive oligomers. LLPS seems however necessary and sufficient to 432 convert these oligomers into ThT-active species and eventually amyloid fibers, as shown by the direct correlation between ThT fluorescence and droplet amount (Figure 3C).

Finally, we demonstrate that the disease-associated mutation P301L promotes both LLPS-HS and amyloid formation (Figure 6). Conversely, P301L mutation has no influence on the formation of 437 LLPS-ED [38]. Therefore, we can conclude that P301L mutation promotes intermolecular 438 hydrophobic interactions that drives LLPS-HS. Furthermore, the correlation plots shown in Figure $4393 \mathrm{C}$ and Figure 6-figure supplement 2C show that at similar droplet quantity, P301L mutant will 440 form greater quantity of ThT active species, and does so more rapidly than tau WT. The 441 aggregation-promoting effect of P301L is well known and elegant explanations have been 442 suggested recently [69, p. 201]. Here, we show that the P301L mutation exerts two seemingly 443 independent effects that both ultimately increase aggregation: (i) It promotes local hydrophobic 444 interactions, thereby promoting hydrophobically-driven phase separation, which in turn triggers 
aggregation and (ii) promotes aggregation-prone conformations, in particular by exposing amyloidogenic segments PHF6(*), independently of LLPS formation. Other disease-related mutations might not combine these effects, because they do not modulate the accessibility of the hydrophobic PHF6(*) segments, explaining why P301L is particularly potent at promoting fibril formation under wide experimental conditions, from in vitro, in cells and in vivo mouse models.

In conclusion, this study uncovers that there is a clear cause-effect relationship between LLPS and aggregation of tau, only when LLPS is driven by hydrophobic association of tau that we found to be preceded by dehydration of its interfacial water. Different physical or biological factors can drive hydrophobically driven LLPS. While this study showcases the principle by relying on dehydration-promoting salting-out mechanisms induced at high $[\mathrm{NaCl}]$ concentration, we posit that hydrophobically driven LLPS can be exerted by many other factors of pathological significance, including the posttranslational modification of tau that can mute the strong positive surface charge density from the abundant lysine residues on tau, e.g. by acetylation and phosphorylation, or enhanced intracellular osmotic crowding pressure, as well as certain pathological mutations of tau, with P301L representing one prominent example. Hence, the liquid condensed state of tau that can be seen in vitro and intra cellularly may be a mediating mechanism by which tau can be sequestered and initially protected from aggregation if LLPS can be reversed, or is inadvertently and irreversibly on pathway towards a pathological state.

\section{Acknowledgements}

465 The authors acknowledge support for the studies of LLPS mechanisms from the National Institutes

466 of Health (NIH) (grant R01AG056058 (S.H.) and support of the Tau consortium of the Rainwater 467 foundation for SH and KSK to study the effect of LLPS on tau pathology. We acknowledge support 468 for the synthesis of BODIPY-NHS dye from the National Science Foundation (DMR-1922042). 469 We acknowledge the use of the NRI-MCDB Microscopy Facility and the Resonant Scanning 470 Confocal supported by NSF MRI grant DBI-1625770 at UC, Santa Barbara. We acknowledge the 471 use of the MRL Shared Experimental Facilities which are supported by the MRSEC Program of 472 the NSF under Award No. DMR 1720256; a member of the NSF-funded Materials Research 473 Facilities Network (www.mrfn.org) 


\section{Materials and Methods}

\section{6 \\ Tau proteins expression and purification}

477

478

479

480

481

482

483

484

485

486

487

488

489

490

491

492

493

494

495

496

497

498

499

500

501

502

503

504

505

506

507

508

509

510

511

512

513

514

515

516

517
Both full length human tau $(2 \mathrm{~N} 4 \mathrm{R})$ and its $\mathrm{N}$-terminal truncated, microtubule binding domain contained variant tau187 (residues 255-441 with a His-tag at the N-terminus) were used for in vitro studies. The cloning, expression, and purification of both $2 \mathrm{~N} 4 \mathrm{R}$ and tau 187 have been previously described [71], [72]. The cysteine-free variants of both 2N4R and tau187 (C291SC322S) were generated via site-direct mutagenesis.

E. coli BL21 (DE3) cells previously transfected were cultured from frozen glycerol stock overnight in $10 \mathrm{~mL}$ luria broth (LB) which was used to inoculate $1 \mathrm{~L}$ of fresh LB. Culturing and inoculation were performed at $37^{\circ} \mathrm{C}$ with shaking of $200 \mathrm{rpm}$. At OD600 of 0.6-0.8. After cell growth, 2N4R culture was incubated with $500 \mathrm{mM} \mathrm{NaCl}$ and $10 \mathrm{mM}$ betaine before inducing expression. Both 2N4R and Tau187 variant expressions were induced by incubation with $1 \mathrm{mM}$ isopropylß-Dthiogalactoside (Sigma Aldrich) for 2-3 h. Cells were harvested by centrifugation for $10 \mathrm{~min}$ at $6000 \times \mathrm{g}$ (Beckman J-10; Beckman Instruments, Inc.), and the pellets were stored at $-20{ }^{\circ} \mathrm{C}$ until further use.

Cell pellets were resuspended in lysis buffer (Tris- $\mathrm{HCl} \mathrm{pH}$ 7.4, $100 \mathrm{mM} \mathrm{NaCl}, 0.5 \mathrm{mM}$ DTT, 0.1 mM EDTA, 1mM PMSF) with 1 Pierce protease inhibitor tablet (Thermo Fisher, EDTA-Free). Lysis was initiated by the addition of lysozyme $(2 \mathrm{mg} / \mathrm{ml})$, DNase $(20 \mu \mathrm{g} / \mathrm{ml})$, and $\mathrm{MgCl}_{2}(10 \mathrm{mM})$ and incubated on an orbital shaker for $30 \mathrm{~min}$ in room temperature. Lysate was then heated to $65{ }^{\circ} \mathrm{C}$ for $13 \mathrm{~min}$, cooled on ice for $20 \mathrm{~min}$ and then centrifuged to remove the precipitant. The supernatant was loaded onto a Ni-NTA agarose column pre-equilibrated with wash buffer A (20 $\mathrm{mM}$ sodium phosphate $\mathrm{pH}$ 7.0, $500 \mathrm{mM} \mathrm{NaCl}, 10 \mathrm{mM}$ imidazole, $100 \mu \mathrm{M}$ EDTA). The column was then washed with $20 \mathrm{ml}$ of buffer A, $15 \mathrm{ml}$ buffer B (20 mM sodium phosphate $\mathrm{pH} 7.0,1 \mathrm{M}$ $\mathrm{NaCl}, 20 \mathrm{mM}$ imidazole, $0.5 \mathrm{mM}$ DTT, $100 \mu \mathrm{M}$ EDTA). Purified tau was eluted with buffer C (20 $\mathrm{mM}$ sodium phosphate $\mathrm{pH}$ 7.0, $0.5 \mathrm{mM}$ DTT, $100 \mathrm{mM} \mathrm{NaCl}$ ) supplemented with varying amounts of imidazole increasing from $100 \mathrm{mM}$ to $300 \mathrm{mM}$. The protein was then concentrated via centrifugal filters (MWCO $10 \mathrm{kDa}$; Millipore Sigma) and the buffer was exchanged into final buffer by PD-10 desalting column (GE Healthcare). The final protein concentration was determined by UV-Vis absorption at $274 \mathrm{~nm}$ using an extinction coefficient of $2.8 \mathrm{~cm}-1 \mathrm{mM}-1$ for tau 187 and $5.8 \mathrm{~cm}-1 \mathrm{mM}-1$ for 2N4R, calculated from absorption of Tyrosine [3]. Same purification protocols were used for both $2 \mathrm{~N} 4 \mathrm{R}$ and tau 187.

\section{Bright field microscopy and TEM imaging}

To confirm the occurrence of LLPS and the formation of liquid droplets, bright field microscope images were taken immediately after sample preparation, using an inverted compound microscope (Zeiss Axtrovert 200M).

For TEM imaging $10 \mu \mathrm{L}$ of recombinant tau fibril samples were applied to copper grid (Electron Microscopy Science, FCF-300-Cu) cleaned with plasma for 20 s. Samples were stained with 10 
$\mu \mathrm{L} 1.5 \mathrm{w} / \mathrm{v} \%$ uranyl acetate. Grids were then imaged with a JEOL JEM-1230 (JEOL USA, Inc) at the indicated magnifications.

\section{Turbidity measurements}

Turbidity of samples at varying $[\mathrm{NaCl}]$ were represented by OD500 measured using a plate reader (Bio-Tek Synergy 2) and 384-well microplate (Corning 3844). To minimize the inference of ThT fluorescence and tau fibril scattering, averaged absorbance of the initial $10 \mathrm{~min}$ where ThT fluorescence is below $10 \%$ was used as turbidity reading.

Turbidity of samples at ramping temperatures were represented by OD500 measured using Jasco J1500 CD Spectrometer (JASCO Inc.) equipped with temperature controller and spectrophotometer. $120 \mu \mathrm{L}$ of tau in designated conditions were prepared in a $100 \mu \mathrm{L}$ cuvette (Starna Scientific Ltd) and kept at $4{ }^{\circ} \mathrm{C}$ for $5 \mathrm{~min}$ before cycling. Heating and cooling temperatures were ramped at $1{ }^{\circ} \mathrm{C} / \mathrm{min}$ while OD500 was monitored.

\section{ThT assay and half time analysis}

LLPS samples were prepared onto a 384-well microplate with additional $1 \mu \mathrm{M}$ Thioflavin T. ThT fluorescence was read from a plate reader (Bio-Tek Synergy 2, excitation 440/30, emission 485/20, number of flash 16). Data was processed using $\mathrm{R}$ package gen5helper following the previous reported methods to reduce artifacts caused by the lamp fluctuation [38].

We followed the previously reported methods [38] to extract the half time and maximum ThT fluorescence, by scaling ThT fluorescence to 0 to 1 and fitting to a sigmoid function,

$$
y=\frac{A}{1+\exp \left(-k\left(t-t_{1 / 2}\right)\right)}
$$

where the fitted $\mathrm{A}$ and $\mathrm{t}_{1 / 2}$ were used as maximum ThT fluorescence and half time, respectively. Fitting results were filtered by $\mathrm{p}<0.05$ to ensure a good fit.

\section{Synthesis of BODIPY-NHS Dye}

BODIPY-NHS was synthesized as previously described with few modifications [55]. A brief description for the synthesis of BODIPY-NHS dye is provided in Figure 2-figure supplement 3 and full details can be found at DOI:10.6084/m9.figshare.12746756. BODIPY-NHS can be synthesized in six steps starting from 4-hydroxybenzaldehyde. In the first step, etherification of 4hydroxybenzaldehyde by potassium carbonate and ethyl 4-bromobutyrate afforded ethyl 4-(4formylphenoxy)butyrate (1) in $98 \%$ yield. Then, hydrolysis with potassium hydroxide provided 44(formyl-phenoxy)butyric acid (2) in $96 \%$ yield. In order to overcome solubility issues that the carboxylic acid intermediate presents in pyrrole, used in step 4, it was converted to the corresponding silyl ester. It reacted with tert-butyl(chloro)diphenylsilane in a reaction promoted by triethyl amine and 4-dimethylaminopyridine affording tert-butyldiphenylsilyl 4-(4formylphenoxy)butanoate (3) in 68\% yield. The aldehyde (3) was then treated with TFA (12 mol\%) and an excess of pyrrole under solvent-free conditions providing the dipyrromethane (4) in $66 \%$ 
$\mathrm{BF}_{3}$. OEt2 and triethylamine lead to BODIPY-COOH in $36 \%$ yield. Steglich esterification with Nhydroxysuccinimide yielded BODIPY-NHS (64\% yield).

\section{Conjugation of BODIPY-NHS to 2N4R}

2N4R protein was diluted to a concentration of $20 \mu \mathrm{M}$ in a PBS buffer. $20 \mathrm{mM}$ BODIPY dissolved in DMSO was added to the protein to a final concentration of $20 \mu \mathrm{M}$. The solution was stirred at room temperature for 4 hours and any unreacted BODIPY-NHS was quenched by adding a solution of excess glycine. The unreacted dye was separated from the conjugated protein with a $7 \mathrm{k}$ MWCO Zeba ${ }^{\mathrm{TM}}$ Spin Desalting Column equilibrated with a $20 \mathrm{mM}$ HEPES buffer, $\mathrm{pH}=7.0$. The conjugated protein was concentrated in an $3 \mathrm{~K}$ MWCO Amicon Ultra Spin Column to a final concentration of approximately $500 \mu \mathrm{M}$. Protein concentration was determined with a Pierce Assay, while the amount of conjugated dye present was calculated by measuring its absorbance at $510 \mathrm{nM}$. Typical labeling reactions gave a 1:1 labeling efficiency.

\section{Measurement and Analysis of FLIM Spectra}

All FLIM measurements were made on a Leica SP8 FALCON Resonant Scanning Confocal Microscope equipped with TCSPC. Images were recorded such that each pixel had a minimum of 1000 photons collected. All data analysis was performed with FLIMFIT [73]. As observed previously [58], lifetime decay curves required multiexponential fits. We found that the higher lifetime component of a 2-exponential fit tracked well with control viscosity measurements made in varying glycerol concentrations and as such it was used for all subsequent analysis.

\section{CwEPR and lineshape analysis}

In this report, we used four variants of tau187 for cwEPR, ODNP and ESEEM studies: tau187/C291S/C322S/S303C, tau187/C291S/C322S/G313C, tau187/C291S, tau187/C291S/C322S/S404C, with one cysteine at site 303, 313, 322 and 404, respectively.

We followed the previous reported methods [38] for spin labeling, cwEPR acquisition, and lineshape analysis. For spin labeling, the freshly eluted variants above were replaced in $20 \mathrm{mM}$ HEPES pH 7.0, using a PD-10 desalting column (GE Healthcare). Protein after PD-10 was labeled overnight at $4{ }^{\circ} \mathrm{C}$ by immediately mixing with a 10 -fold molar excess of the spin label (1-oxyl2,2,5,5-tetramethylpyrroline-3-methyl) methanethiosulfonate (MTSL; Toronto Research Chemicals), resulting in spin labelled tau at designated site. Excess label was removed using PD10. The protein was concentrated using centrifugal filter (MWCO $10 \mathrm{kDa}$; Amicon) and the final protein concentration was determined by UV-Vis absorption at $274 \mathrm{~nm}$ as mentioned above. Cystein-free non-labeled tau187C291SC322S was used in order to achieve spin dilution.

$\mathrm{Cw}$ EPR measurements were carried out using a X-band spectrometer operating at $9.8 \mathrm{GHz}$ (EMX; Bruker Biospin, Billerica, MA) and a dielectric cavity (ER 4123D; Bruker Biospin, Billerica, MA). Spin labeled tau187 was mixed with tau187C291SC322S at 1:4 molar ratio to reach $20 \%$ spin labeling. A sample of $4.0 \mu \mathrm{l}$ volume was loaded into a quartz capillary (CV6084; VitroCom) and sealed at both ends with critoseal, and then placed in the dielectric cavity for measurements. $\mathrm{Cw}$ 
EPR spectra were acquired by using $1.8 \mathrm{~mW}$ of microwave power, 0.5 gauss modulation amplitude, 100 gauss sweep width, and 8-64 scans for signal averaging.

The recorded cw EPR spectra were fit with simulation using models with incremental complexity. EPR simulation and fitting were performed using MultiComponent, a program developed by Christian Altenbach (University of California, Los Angeles). For all spectra fitting, the magnetic tensors $\mathrm{A}$ and $\mathrm{g}$ were fixed and used as constraints as previously reported [74]. These values are $\mathrm{A}_{\mathrm{xx}}=6.2 \mathrm{G}, \mathrm{A}_{\mathrm{yy}}=5.9 \mathrm{G}, \mathrm{A}_{z z}=37.0 \mathrm{G}$, and $\mathrm{g}_{\mathrm{xx}}=2.0078, \mathrm{~g}_{\mathrm{yy}}=2.0058$, and $\mathrm{g}_{z z}=2.0022$. For soluble tau and tau at $[\mathrm{NaCl}]<2.0 \mathrm{M}$, the cw EPR spectra of all four spin-labeled tau variants were best fitted with a single isotropic component simulation and the rotational diffusion constant $(\mathrm{R})$ can be extracted. The rotation correlation time $\tau_{\mathrm{R}}$ was calculated using $\tau_{\mathrm{R}}=1 /(6 \mathrm{R})$. For tau at 2.0 $\mathrm{M}<[\mathrm{NaCl}]<4.0 \mathrm{M}$, the single isotropic component simulation failed to capture the lineshape (Figure 5-figure supplement 1A). A second isotropic component was added to increase the complexity of the model. The second isotropic component were set to be identical to the first component, except with an independent $\mathrm{R}$ value. The fitting parameters were limited at a minimum, which includes the population, $\mathrm{p}$ and the rotational diffusion constants of the first and second isotropic component $\mathrm{R}_{1}$ and $\mathrm{R}_{2}$. For tau at $[\mathrm{NaCl}]>4.0 \mathrm{M}$, both the signal and double isotropic component simulation failed to capture the lineshape (Figure 5-figure supplement 1B). Anisotropic parameters were added to the second components and subjected to fit. The fitting parameters include $\mathrm{p}, \mathrm{R}_{1}, \mathrm{R}_{2}$, and the added parameters for the second components including the tilt angle of the diffusion tensor $\alpha \mathrm{D}, \beta \mathrm{D}$, orienting potentials $\mathrm{c}_{20}, \mathrm{c} 22, \mathrm{c} 40$, c44. Fitted spectra and parameters of all four sites at different $[\mathrm{NaCl}]$ are shown in Figure 5-figure supplement 2 and Figure 5-source data 1 , respectively.

\section{Overhauser dynamic nuclear polarization (ODNP)}

We used the aforementioned four spin labeled tau187 variants (singly labeled on site 303, 313, 322 and 404, respectively), and followed previously reported methods for ODNP experiments [30]. $100 \mu \mathrm{M}$ spin labeled tau187 was mixed with designated concentration of $\mathrm{NaCl}$. Immediately after sample preparation, $3.5 \mu \mathrm{L}$ of the samples were loaded into quartz capillaries of $0.6 \mathrm{~mm}$ ID $\times 0.84 \mathrm{~mm}$ OD (Vitrocom, New Jersey, USA), and two ends of the tubes were sealed with Critoseal and beeswax respectively. ODNP experiments were performed using a Bruker EMXPlus spectrometer and a Bruker Avance III NMR console (Bruker, Massachusetts, USA). The capillary tube was mounted on a home-built NMR probe with a U-shaped NMR coil, and was set in a Bruker ER 4119HS-LC sensitivity cavity. Samples were irradiated at $9.8 \mathrm{GHz}$ with the center field set at $3484 \mathrm{G}$ and sweep width of $120 \mathrm{G}$. Dry air was kept at temperature of $18{ }^{\circ} \mathrm{C}$ and purged through the NMR probe during all measurements. ODNP data was analyzed using customized python script WorkupODNP and ODNPLab, available on https://www.github.com. Theory of ODNP and details in the experiment are previously reported in other studies [63], [64].

653

\section{3-Pulse Electron Spin Echo Envelope Modulation (ESEEM)}

We followed previously reported methods for ESEEM measurements [75]. ESEEM measurements were performed at X-Band on an ELEXSYS E580 spectrometer at $80 \mathrm{~K}$ with an Oxford instruments cryostat (CF935P) and temperature controller (ITC503). A Bruker MS3 resonator was maximally overcoupled to a Q-factor of approximately 100 for these measurements. Immediately 
654 after sample preparation, $35 \mu \mathrm{L}$ of sample was loaded into a $3 \mathrm{~mm}$ OD quartz EPR tube and flash 655 frozen in liquid nitrogen before being inserted into the resonator. Spin labeled tau187 at site 313 656 was used for measurement. All experiments were carried out in deuterated $20 \mathrm{mM}$ HEPES with $65720 \mathrm{wt} \%$ Ficoll.

The ESEEM data was fit by 5th order polynomial to correct the background decay, hamming windowed, zero-filled and Fourier-transformed, following reported method [75]. We interpreted the reduce of the the intensity of the deuterium peak as a result of dehydration near the site 313 . Raw data was shown in Figure 4-figure supplement 2.

\section{Estimate Unbound Tau Concentration}

670

Assuming tau binding to microtubule has a stoichiometry of 1:1 between tau molecule and tubulin monomer, regardless of different types of tubulin monomers, the dissociation process can be expressed as

$$
[\text { tau } * \text { tubulin }] \rightarrow[\text { tau }]+[\text { tubulin }]
$$

where [tau*tubulin] and [tau] represent the bounded and unbounded tau concentration, respectively. Knowing 


\section{References}

679

680

681

682

683

684

685

686

687

688

689

690

691

692

693

694

695

696

697

698

699

700

701

702

703

704

705

706

707

708

709

710

711

712

713

714

715

716

717

718

719

720
[1] T. Guo, W. Noble, and D. P. Hanger, "Roles of tau protein in health and disease," Acta Neuropathol. (Berl.), vol. 133, no. 5, pp. 665-704, 2017, doi: 10.1007/s00401-017-1707-9.

[2] J. Avila, J. J. Lucas, M. Perez, and F. Hernandez, "Role of tau protein in both physiological and pathological conditions," Physiol. Rev., vol. 84, no. 2, pp. 361-384, Apr. 2004, doi: 10.1152/physrev.00024.2003.

[3] J. Q. Trojanowski, T. Schuck, M. L. Schmidt, and V. M. Lee, "Distribution of tau proteins in the normal human central and peripheral nervous system," J. Histochem. Cytochem. Off. J. Histochem. Soc., vol. 37, no. 2, pp. 209-215, Feb. 1989, doi: 10.1177/37.2.2492045.

[4] M. Ackmann, H. Wiech, and E. Mandelkow, "Nonsaturable Binding Indicates Clustering of Tau on the Microtubule Surface in a Paired Helical Filament-like Conformation," J. Biol. Chem., vol. 275, no. 39, pp. 30335-30343, Sep. 2000, doi: 10.1074/jbc.M002590200.

[5] G. Hiller and K. Weber, "Radioimmunoassay for tubulin: a quantitative comparison of the tubulin content of different established tissue culture cells and tissues," Cell, vol. 14, no. 4, pp. 795-804, Aug. 1978, doi: 10.1016/0092-8674(78)90335-5.

[6] H. Ksiezak-Reding, L. I. Binder, and S. H. Yen, "Immunochemical and biochemical characterization of tau proteins in normal and Alzheimer's disease brains with Alz 50 and Tau-1.," J. Biol. Chem., vol. 263, no. 17, pp. 7948-7953, Jun. 1988, Accessed: May 31, 2019. [Online]. Available: http://www.jbc.org/content/263/17/7948.

[7] N. Gustke, B. Trinczek, J. Biernat, E.-M. Mandelkow, and E. Mandelkow, "Domains of tau Protein and Interactions with Microtubules," Biochemistry, vol. 33, no. 32, pp. 9511-9522, Oct. 1994, doi: 10.1021/bi00198a017.

[8] T. Arendt, J. T. Stieler, and M. Holzer, "Tau and tauopathies," Brain Res. Bull., vol. 126, pp. 238-292, Sep. 2016, doi: 10.1016/j.brainresbull.2016.08.018.

[9] C. P. Brangwynne, P. Tompa, and R. V. Pappu, "Polymer physics of intracellular phase transitions," Nat. Phys., vol. 11, no. 11, pp. 899-904, Nov. 2015, doi: 10.1038/nphys3532.

[10] A. A. Hyman, C. A. Weber, and F. Jülicher, "Liquid-Liquid Phase Separation in Biology," Annu. Rev. Cell Dev. Biol., vol. 30, no. 1, pp. 39-58, 2014, doi: 10.1146/annurev-cellbio100913-013325.

[11] S. Alberti and S. Carra, "Quality Control of Membraneless Organelles," J. Mol. Biol., vol. 430, no. 23, pp. 4711-4729, Nov. 2018, doi: 10.1016/j.jmb.2018.05.013.

[12] J. A. Toretsky and P. E. Wright, "Assemblages: Functional units formed by cellular phase separation," J Cell Biol, vol. 206, no. 5, pp. 579-588, Sep. 2014, doi: 10.1083/jcb.201404124.

[13] A. Molliex et al., "Phase Separation by Low Complexity Domains Promotes Stress Granule Assembly and Drives Pathological Fibrillization," Cell, vol. 163, no. 1, pp. 123-133, Sep. 2015, doi: 10.1016/j.cell.2015.09.015.

[14] Y. R. Li, O. D. King, J. Shorter, and A. D. Gitler, "Stress granules as crucibles of ALS pathogenesis," J Cell Biol, vol. 201, no. 3, pp. 361-372, Apr. 2013, doi: 10.1083/jcb.201302044.

[15] T. Murakami et al., "ALS/FTD Mutation-Induced Phase Transition of FUS Liquid Droplets and Reversible Hydrogels into Irreversible Hydrogels Impairs RNP Granule Function," Neuron, vol. 88, no. 4, pp. 678-690, Nov. 2015, doi: 10.1016/j.neuron.2015.10.030. 
[16] A. Patel et al., "A Liquid-to-Solid Phase Transition of the ALS Protein FUS Accelerated by Disease Mutation," Cell, vol. 162, no. 5, pp. 1066-1077, Aug. 2015, doi: 10.1016/j.cell.2015.07.047.

[17] Y. Chen and T. J. Cohen, "Aggregation of the nucleic acid-binding protein TDP-43 occurs via distinct routes that are coordinated with stress granule formation," J. Biol. Chem., vol. 294, no. 10, pp. 3696-3706, 08 2019, doi: 10.1074/jbc.RA118.006351.

[18] S. Ray et al., "Liquid-liquid phase separation and liquid-to-solid transition mediate $\alpha$ synuclein amyloid fibril containing hydrogel formation," bioRxiv, p. 619858, Apr. 2019, doi: 10.1101/619858.

[19] S. Ambadipudi, J. Biernat, D. Riedel, E. Mandelkow, and M. Zweckstetter, "Liquid-liquid phase separation of the microtubule-binding repeats of the Alzheimer-related protein Tau," Nat. Commun., vol. 8, no. 1, p. 275, Aug. 2017, doi: 10.1038/s41467-017-00480-0.

[20] S. Wegmann et al., "Tau protein liquid-liquid phase separation can initiate tau aggregation," EMBO J., vol. 37, no. 7, Apr. 2018, doi: 10.15252/embj.201798049.

[21] X. Zhang et al., "RNA Stores Tau Reversibly in Complex Coacervates," bioRxiv, p. 111245, Feb. 2017, doi: 10.1101/111245.

[22] Y. Lin et al., "Narrow equilibrium window for complex coacervation of tau and RNA under cellular conditions," eLife, vol. 8, p. e42571, Apr. 2019, doi: 10.7554/eLife.42571.

[23] A. Hernández-Vega et al., "Local Nucleation of Microtubule Bundles through Tubulin Concentration into a Condensed Tau Phase," Cell Rep., vol. 20, no. 10, pp. 2304-2312, Sep. 2017, doi: 10.1016/j.celrep.2017.08.042.

[24] J. C. Ferreon et al., "Acetylation Disfavors Tau Phase Separation,” Int. J. Mol. Sci., vol. 19, no. 5, May 2018, doi: 10.3390/ijms 19051360.

[25] A. Majumdar, P. Dogra, S. Maity, and S. Mukhopadhyay, "Liquid-Liquid Phase Separation Is Driven by Large-Scale Conformational Unwinding and Fluctuations of Intrinsically Disordered Protein Molecules," J. Phys. Chem. Lett., vol. 10, no. 14, pp. 3929-3936, Jul. 2019, doi: 10.1021/acs.jpclett.9b01731.

[26] S. Park, S. Kim, Y. Jho, and D. S. Hwang, "Cation- $\pi$ Interactions and Their Contribution to Mussel Underwater Adhesion Studied Using a Surface Forces Apparatus: A Mini-Review," Langmuir, Aug. 2019, doi: 10.1021/acs.langmuir.9b01976.

[27] S. F. Banani, H. O. Lee, A. A. Hyman, and M. K. Rosen, "Biomolecular condensates: organizers of cellular biochemistry," Nat. Rev. Mol. Cell Biol., vol. 18, no. 5, pp. 285-298, May 2017, doi: 10.1038/nrm.2017.7.

[28] H. J. Dyson, P. E. Wright, and H. A. Scheraga, "The role of hydrophobic interactions in initiation and propagation of protein folding," Proc. Natl. Acad. Sci., vol. 103, no. 35, pp. 13057-13061, Aug. 2006, doi: 10.1073/pnas.0605504103.

[29] B. Gabryelczyk et al., "Hydrogen bond guidance and aromatic stacking drive liquid-liquid phase separation of intrinsically disordered histidine-rich peptides," Nat. Commun., vol. 10, no. 1, pp. 1-12, Nov. 2019, doi: 10.1038/s41467-019-13469-8.

[30] S. Park et al., "Dehydration entropy drives liquid-liquid phase separation by molecular crowding," Commun. Chem., vol. 3, no. 1, pp. 1-12, Jun. 2020, doi: 10.1038/s42004-0200328-8.

[31] S. L. Crick, K. M. Ruff, K. Garai, C. Frieden, and R. V. Pappu, "Unmasking the roles of Nand C-terminal flanking sequences from exon 1 of huntingtin as modulators of polyglutamine aggregation," Proc. Natl. Acad. Sci. U. S. A., vol. 110, no. 50, pp. 2007520080, Dec. 2013, doi: 10.1073/pnas.1320626110. 
[32] S. Elbaum-Garfinkle et al., "The disordered P granule protein LAF-1 drives phase separation into droplets with tunable viscosity and dynamics," Proc. Natl. Acad. Sci. U. S. A., vol. 112, no. 23, pp. 7189-7194, Jun. 2015, doi: 10.1073/pnas.1504822112.

[33] S. Boyko, X. Qi, T.-H. Chen, K. Surewicz, and W. K. Surewicz, "Liquid-liquid phase separation of tau protein: The crucial role of electrostatic interactions," J. Biol. Chem., p. jbc.AC119.009198, May 2019, doi: 10.1074/jbc.AC119.009198.

[34] V. Singh, L. Xu, S. Boyko, K. Surewicz, and W. K. Surewicz, "Zinc promotes liquid-liquid phase separation of tau protein," J. Biol. Chem., p. jbc.AC120.013166, Mar. 2020, doi: 10.1074/jbc.AC120.013166.

[35] P. Li et al., "Phase transitions in the assembly of multivalent signalling proteins," Nature, vol. 483, no. 7389, pp. 336-340, Mar. 2012, doi: 10.1038/nature10879.

[36] S. Kim et al., "Complexation and coacervation of like-charged polyelectrolytes inspired by mussels,” Proc. Natl. Acad. Sci., vol. 113, no. 7, pp. E847-E853, Feb. 2016, doi: 10.1073/pnas.1521521113.

[37] T. J. Nott et al., "Phase Transition of a Disordered Nuage Protein Generates Environmentally Responsive Membraneless Organelles," Mol. Cell, vol. 57, no. 5, pp. 936947, Mar. 2015, doi: 10.1016/j.molcel.2015.01.013.

[38] Y. Lin, Y. Fichou, Z. Zeng, N. Y. Hu, and S. Han, "Electrostatically Driven Complex Coacervation and Amyloid Aggregation of Tau Are Independent Processes with Overlapping Conditions," ACS Chem. Neurosci., Jan. 2020, doi: 10.1021/acschemneuro.9b00627.

[39] W. Kunz, J. Henle, and B. W. Ninham, "'Zur Lehre von der Wirkung der Salze' (About the science of the effect of salts): Franz Hofmeister's historical papers," Curr. Opin. Colloid Interface Sci., vol. 9, no. 1-2, pp. 19-37, 2004, Accessed: Jul. 03, 2020. [Online]. Available: https://epub.uni-regensburg.de/24565/.

[40] Y. Zhang and P. S. Cremer, "Interactions between macromolecules and ions: the Hofmeister series," Curr. Opin. Chem. Biol., vol. 10, no. 6, pp. 658-663, Dec. 2006, doi: 10.1016/j.cbpa.2006.09.020.

[41] R. A. Curtis, C. Steinbrecher, M. Heinemann, H. W. Blanch, and J. M. Prausnitz, "Hydrophobic forces between protein molecules in aqueous solutions of concentrated electrolyte," Biophys. Chem., vol. 98, no. 3, pp. 249-265, Aug. 2002, doi: 10.1016/S03014622(02)00071-6.

[42] T. Ghosh, A. Kalra, and S. Garde, "On the Salt-Induced Stabilization of Pair and Manybody Hydrophobic Interactions," J. Phys. Chem. B, vol. 109, no. 1, pp. 642-651, Jan. 2005, doi: 10.1021/jp0475638.

[43] M. K. Braun et al., "Reentrant Phase Behavior in Protein Solutions Induced by Multivalent Salts: Strong Effect of Anions Cl- Versus NO3-," J. Phys. Chem. B, vol. 122, no. 50, pp. 11978-11985, Dec. 2018, doi: 10.1021/acs.jpcb.8b10268.

[44] Y. Zhang and P. S. Cremer, "The inverse and direct Hofmeister series for lysozyme," Proc. Natl. Acad. Sci., vol. 106, no. 36, pp. 15249-15253, Sep. 2009, doi: 10.1073/pnas.0907616106.

[45] G. Krainer et al., "Reentrant liquid condensate phase of proteins is stabilized by hydrophobic and non-ionic interactions," bioRxiv, p. 2020.05.04.076299, May 2020, doi: 10.1101/2020.05.04.076299. 
[46] S. H. Scheres, W. Zhang, B. Falcon, and M. Goedert, "Cryo-EM structures of tau filaments," Curr. Opin. Struct. Biol., vol. 64, pp. 17-25, Oct. 2020, doi: 10.1016/j.sbi.2020.05.011.

[47] S. Kroschwald, S. Maharana, and A. Simon, "Hexanediol: a chemical probe to investigate the material properties of membrane-less compartments," Matters, vol. 3, no. 5, p. e201702000010, May 2017, doi: 10.19185/matters.201702000010.

[48] K. Ribbeck and D. Görlich, "The permeability barrier of nuclear pore complexes appears to operate via hydrophobic exclusion," EMBO J., vol. 21, no. 11, pp. 2664-2671, Jun. 2002, doi: 10.1093/emboj/21.11.2664.

[49] S. S. Patel, B. J. Belmont, J. M. Sante, and M. F. Rexach, "Natively Unfolded Nucleoporins Gate Protein Diffusion across the Nuclear Pore Complex," Cell, vol. 129, no. 1, pp. 83-96, Apr. 2007, doi: 10.1016/j.cell.2007.01.044.

[50] A. D. Gitler, J. Shorter, T. Ha, and S. Myong, "Just Took a DNA Test, Turns Out 100\% Not That Phase," Mol. Cell, vol. 78, no. 2, pp. 193-194, Apr. 2020, doi: 10.1016/j.molcel.2020.03.029.

[51] S. Ambadipudi, J. G. Reddy, J. Biernat, E. Mandelkow, and M. Zweckstetter, "Residuespecific identification of phase separation hot spots of Alzheimer's-related protein tau," Chem. Sci., vol. 10, no. 26, pp. 6503-6507, Jul. 2019, doi: 10.1039/C9SC00531E.

[52] J. N. Israelachvili, Intermolecular and Surface Forces. Academic Press, 2011.

[53] J. S. D'Arrigo, "Screening of membrane surface charges by divalent cations: an atomic representation," Am. J. Physiol., vol. 235, no. 3, pp. C109-117, Sep. 1978, doi: 10.1152/ajpcell.1978.235.3.C109.

[54] Y. Lin, D. S. W. Protter, M. K. Rosen, and R. Parker, "Formation and Maturation of PhaseSeparated Liquid Droplets by RNA-Binding Proteins," Mol. Cell, vol. 60, no. 2, pp. 208219, Oct. 2015, doi: 10.1016/j.molcel.2015.08.018.

[55] M. Kubánková et al., "Probing supramolecular protein assembly using covalently attached fluorescent molecular rotors," Biomaterials, vol. 139, pp. 195-201, Sep. 2017, doi: 10.1016/j.biomaterials.2017.06.009.

[56] J. E. Chambers et al., "An Optical Technique for Mapping Microviscosity Dynamics in Cellular Organelles,” ACS Nano, vol. 12, no. 5, pp. 4398-4407, May 2018, doi: 10.1021/acsnano.8b00177.

[57] S.-C. Lee et al., "Fluorescent Molecular Rotors for Viscosity Sensors," Chem. - Eur. J., vol. 24, no. 52, pp. 13706-13718, Sep. 2018, doi: 10.1002/chem.201801389.

[58] M. K. Kuimova, G. Yahioglu, J. A. Levitt, and K. Suhling, "Molecular Rotor Measures Viscosity of Live Cells via Fluorescence Lifetime Imaging," J. Am. Chem. Soc., vol. 130, no. 21, pp. 6672-6673, May 2008, doi: 10.1021/ja800570d.

[59] D. R. Robinson and W. P. Jencks, "The Effect of Concentrated Salt Solutions on the Activity Coefficient of Acetyltetraglycine Ethyl Ester," J. Am. Chem. Soc., vol. 87, no. 11, pp. 2470-2479, Jun. 1965, doi: 10.1021/ja01089a029.

[60] A. Volkov, C. Dockter, T. Bund, H. Paulsen, and G. Jeschke, "Pulsed EPR determination of water accessibility to spin-labeled amino acid residues in LHCIIb," Biophys. J., vol. 96, no. 3, pp. 1124-1141, Feb. 2009, doi: 10.1016/j.bpj.2008.09.047.

[61] J. M. Franck et al., "Probing Water Density and Dynamics in the Chaperonin GroEL Cavity,” J. Am. Chem. Soc., vol. 136, no. 26, pp. 9396-9403, Jul. 2014, doi: 10.1021/ja503501x. 
[62] A. Doll, E. Bordignon, B. Joseph, R. Tschaggelar, and G. Jeschke, "Liquid state DNP for water accessibility measurements on spin-labeled membrane proteins at physiological temperatures," J. Magn. Reson. San Diego Calif 1997, vol. 222, pp. 34-43, Sep. 2012, doi: 10.1016/j.jmr.2012.06.003.

[63] B. D. Armstrong and S. Han, "Overhauser Dynamic Nuclear Polarization To Study Local Water Dynamics,” J. Am. Chem. Soc., vol. 131, no. 13, pp. 4641-4647, Apr. 2009, doi: 10.1021/ja809259q.

[64] J. M. Franck, Y. Ding, K. Stone, P. Z. Qin, and S. Han, “Anomalously Rapid Hydration Water Diffusion Dynamics Near DNA Surfaces,” J. Am. Chem. Soc., vol. 137, no. 37, pp. 12013-12023, Sep. 2015, doi: 10.1021/jacs.5b05813.

[65] T. F. Segawa, M. Doppelbauer, L. Garbuio, A. Doll, Y. O. Polyhach, and G. Jeschke, "Water accessibility in a membrane-inserting peptide comparing Overhauser DNP and pulse EPR methods," J. Chem. Phys., vol. 144, no. 19, p. 194201, May 2016, doi: 10.1063/1.4948988.

[66] W. L. Hubbell and C. Altenbach, "Investigation of structure and dynamics in membrane proteins using site-directed spin labeling," Curr. Opin. Struct. Biol., vol. 4, no. 4, pp. 566573, Jan. 1994, doi: 10.1016/S0959-440X(94)90219-4.

[67] D. E. Budil, S. Lee, S. Saxena, and J. H. Freed, "Nonlinear-Least-Squares Analysis of Slow-Motion EPR Spectra in One and Two Dimensions Using a Modified LevenbergMarquardt Algorithm," J. Magn. Reson. A, vol. 120, no. 2, pp. 155-189, Jun. 1996, doi: 10.1006/jmra.1996.0113.

[68] J. S. Hwang, R. P. Mason, L. P. Hwang, and J. H. Freed, "Electron spin resonance studies of anisotropic rotational reorientation and slow tumbling in liquid and frozen media. III. Perdeuterated 2,2,6,6-tetramethyl-4-piperidone N-oxide and an analysis of fluctuating torques," J. Phys. Chem., vol. 79, no. 5, pp. 489-511, Feb. 1975, doi: $10.1021 / \mathrm{j} 100572 \mathrm{a} 017$.

[69] D. Chen et al., "Tau local structure shields an amyloid-forming motif and controls aggregation propensity," Nat. Commun., vol. 10, no. 1, pp. 1-14, Jun. 2019, doi: 10.1038/s41467-019-10355-1.

[70] G. C. Ruben, T. L. Ciardelli, I. Grundke-Iqbal, and K. Iqbal, "Alzheimer disease hyperphosphorylated tau aggregates hydrophobically," Synapse, vol. 27, no. 3, pp. 208229, 1997, doi: 10.1002/(SICI)1098-2396(199711)27:3<208::AID-SYN7>3.0.CO;2-H.

[71] A. Pavlova, E. R. McCarney, D. W. Peterson, F. W. Dahlquist, J. Lew, and S. Han, "Sitespecific dynamic nuclear polarization of hydration water as a generally applicable approach to monitor protein aggregation," Phys. Chem. Chem. Phys. PCCP, vol. 11, no. 31, pp. 6833-6839, Aug. 2009, doi: 10.1039/b906101k.

[72] D. W. Peterson, H. Zhou, F. W. Dahlquist, and J. Lew, "A soluble oligomer of tau associated with fiber formation analyzed by NMR," Biochemistry, vol. 47, no. 28, pp. 7393-7404, Jul. 2008, doi: 10.1021/bi702466a.

[73] S. C. Warren et al., "Rapid Global Fitting of Large Fluorescence Lifetime Imaging Microscopy Datasets," PLOS ONE, vol. 8, no. 8, p. e70687, Aug. 2013, doi: 10.1371/journal.pone.0070687.

[74] A. Pavlova, C.-Y. Cheng, M. Kinnebrew, J. Lew, F. W. Dahlquist, and S. Han, "Protein structural and surface water rearrangement constitute major events in the earliest aggregation stages of tau," Proc. Natl. Acad. Sci., vol. 113, no. 2, pp. E127-E136, 2016, 
Accessed: Mar. 23, 2016. [Online]. Available: http://www.pnas.org/content/113/2/E127.short.

903

[75] Y. Fichou et al., "Tau-Cofactor Complexes as Building Blocks of Tau Fibrils," Front.

904 Neurosci., vol. 13, 2019, doi: 10.3389/fnins.2019.01339.

905

906 
$908 \quad$ Figure 1

A
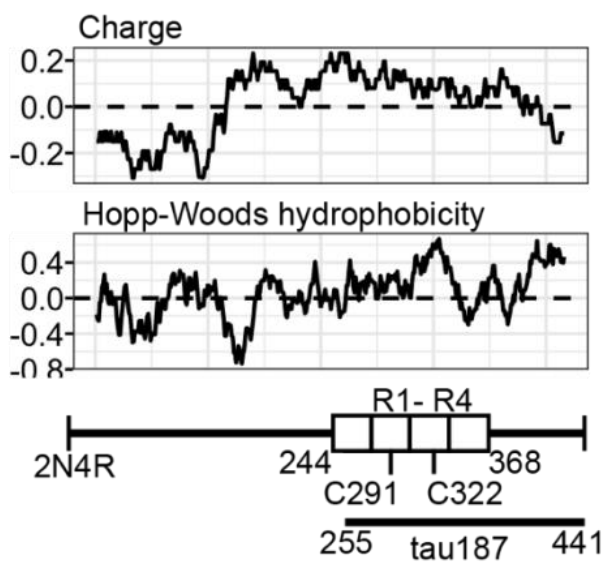

C $100 \mu \mathrm{M}$ tau 187 with $4.75 \mathrm{M} \mathrm{NaCl}$. Scale bar length is $25 \mu \mathrm{m}$.
B

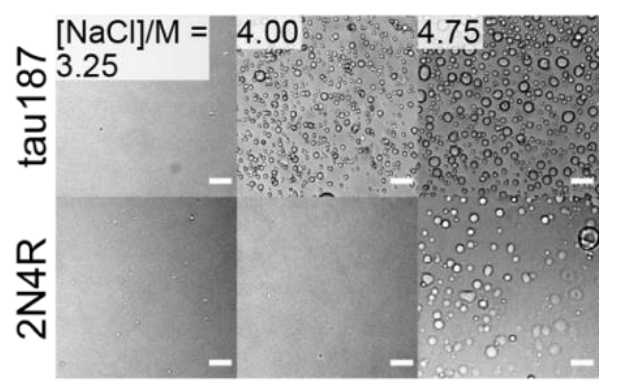

D +RNA low salt high salt

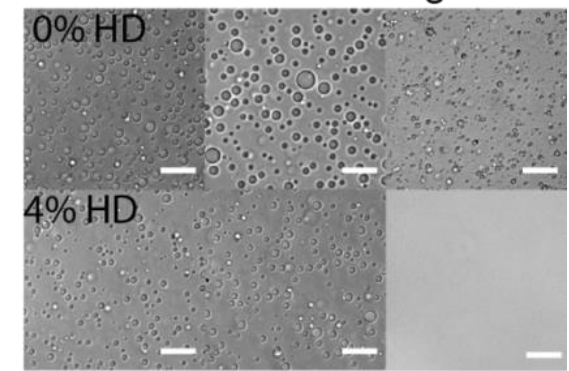

Figure 1 Tau undergoes LLPS at high salt concentration. A. Charge and Hopp-Woods hydrophobicity plot of 2N4R. Data points show the average values of consecutive 25 amino acids. Reference values of Glycine are shown as dashed lines. tau187 fragment (residues 255-441), four repeat domains (R1-R4, 244-368) and two native cysteines (C291, C322) are shown. B. Microscope images of tau187 and 2N4R at varying [NaCl]. $44 \mu \mathrm{M}$ tau187 and $20 \mu \mathrm{M} 2 \mathrm{~N} 4 \mathrm{R}$ were chosen to have same mass concentration. Scale bar length is $25 \mu \mathrm{m}$. C. Droplet merging of tau LLPS-HS. $450 \mu \mathrm{M}$ tau187 with $2.2 \mathrm{M} \mathrm{NaCl}$ was used. Scale bar length is $50 \mu \mathrm{m}$. D. Effects of 4 wt\% 1,6-hexanediol on different types of tau LLPS. Tau-RNA LLPS (+RNA) was prepared with $20 \mu \mathrm{M} 2 \mathrm{~N} 4 \mathrm{R}$ and $40 \mu \mathrm{g} / \mathrm{mL}$ polyU RNA; Tau low salt simple coacervation (low salt) was prepared with $20 \mu \mathrm{M} 2 \mathrm{~N} 4 \mathrm{R}$ at $5 \mathrm{mM} \mathrm{NaCl}$; Tau high salt simple coacervation (high salt) was prepared with 
bioRxiv preprint doi: https://doi.org/10.1101/2020.08.05.237966; this version posted August 6, 2020. The copyright holder for this preprint (which was not certified by peer review) is the author/funder. All rights reserved. No reuse allowed without permission.

922 Figure 1-figure supplement 1.

923

924

925

926

927

928

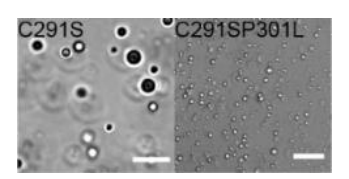

Figure 1-figure supplement 1. Microscope images of LLPS-high salt of various tau constructs. $450 \mu \mathrm{M}$ Tau187C291S with 2.2 M NaCl; $100 \mu \mathrm{M}$ tau187C291SP301L with 3.0 M NaCl. 
Figure 2
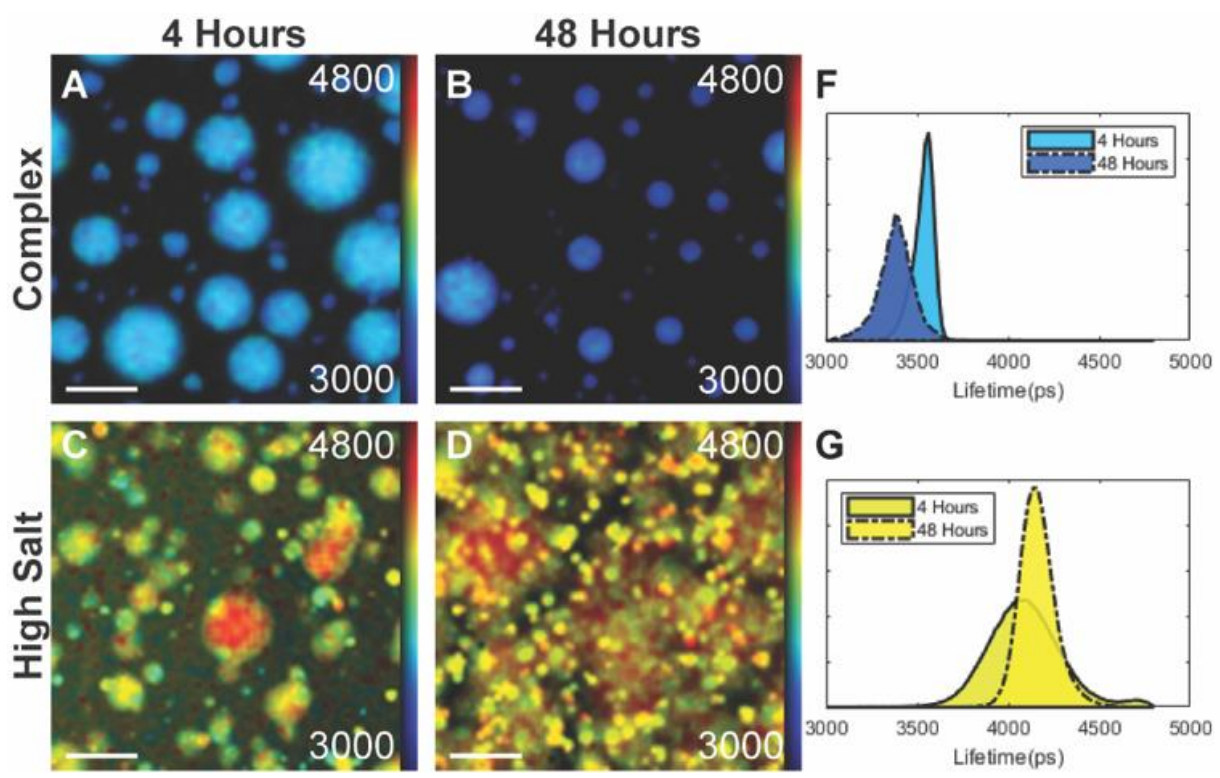

Figure 2 FLIM fits of tau-RNA LLPS-CC (complex), and tau LLPS-high salt (high salt). Fluorescence Lifetime Imaging Measurements were taken at 4 and 48 hours at the same resolution, scale bar $=25 \mu \mathrm{m}$. A-D, Fluorescent microscope images of tau-RNA droplets and tau high salt droplets before (4 hour) and after incubation (48 hour). Individual pixels were fitted with a 2component exponential decay using the FLIMfit software tool developed at Imperial College London (Warren SC et al, PLOS ONE 2013), and the data were visualized using the higher lifetime component. The colors in the fluorescent images and corresponding histograms represent a pseudo color heat map that ranges from 3.0 to $4.8 \mathrm{~ns}$, with blue representing low lifetimes and red representing high lifetimes. F-G. Histograms of fitted results in A-D. The histograms are normalized so that area under the curve is unity. The color under each histogram corresponds to the pseudo color of the heat map at the histograms max value. Solid line $=4$ hours, Dashed line $=$ 48 hours. 


\section{Figure 2-figure supplement 1.}
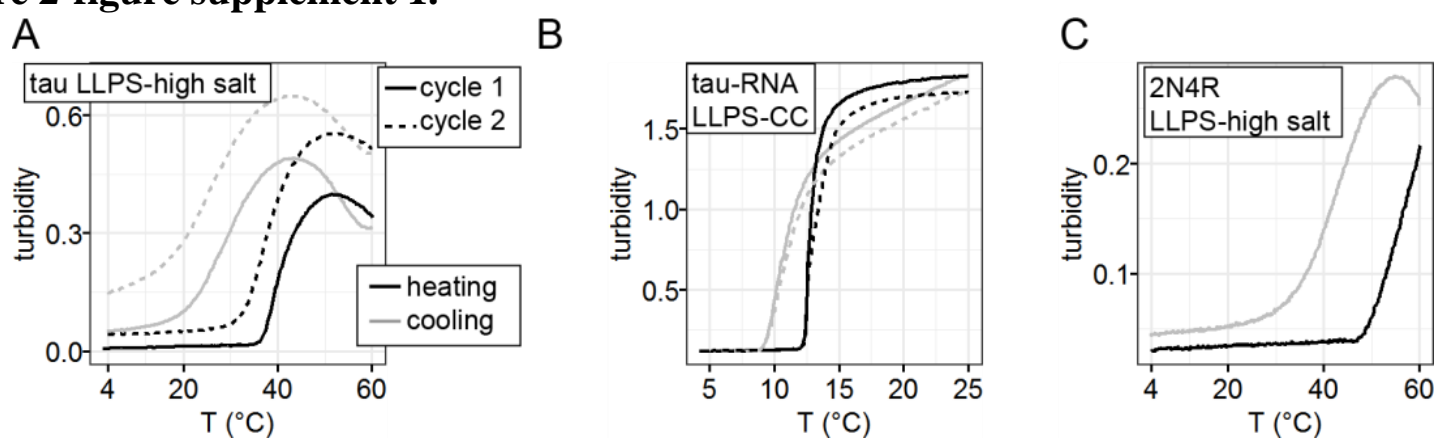

Figure 2-figure supplement 1. Temperature dependence of LLPS-HS. A. turbidity of tau187 LLPS-high salt upon heating-cooling cycles. $34 \mu \mathrm{M}$ tau187 and $3.5 \mathrm{M} \mathrm{NaCl}$ was used. B. turbidity of tau187-RNA LLPS-CC upon heating-cooling cycles. $100 \mu \mathrm{M}$ tau187 with 300 $\mu \mathrm{g} / \mathrm{mL}$ PolyU RNA and $30 \mathrm{mM} \mathrm{NaCl}$ was used. C. turbidity of 2N4R LLPS-high salt upon heating-cooling cycle. $20 \mu \mathrm{M}$ tau with $3.5 \mathrm{M} \mathrm{NaCl}$ was used. Samples were pre-cooled to $4{ }^{\circ} \mathrm{C}$, heated to 25 or $60{ }^{\circ} \mathrm{C}$ then cooled back to $4{ }^{\circ} \mathrm{C}$ (cycle 1, solid lines). A second cycle immediately follows (cycle 2 , dashed lines). The rate of both heating and cooling are $4{ }^{\circ} \mathrm{C} / \mathrm{min}$ in $\mathrm{A}$ and $\mathrm{C}$, and $1{ }^{\circ} \mathrm{C} / \mathrm{min}$ at $\mathrm{B}$. 
Figure 2-figure supplement 2.

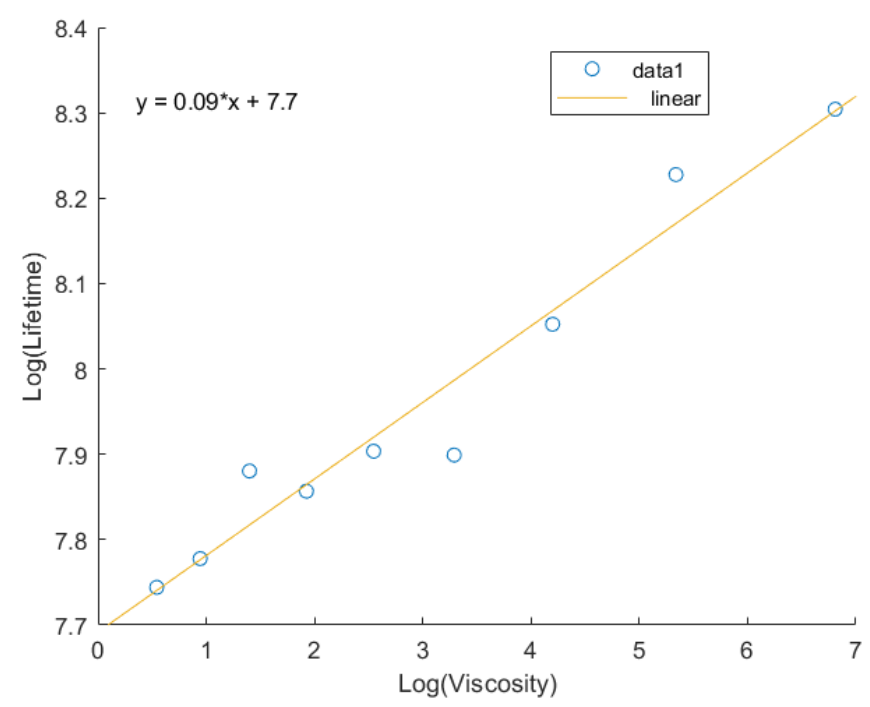

Figure 2-figure supplement 2. Calibration and Fit of Fluorescent Lifetimes to a Glycerol Standard Curve. $4 \mu \mathrm{M}$ 2N4R conjugated the BODIPY was placed in solutions of glycerol concentrations in $\mathrm{ddH}_{2} \mathrm{O}$ ranging from $20-100 \%$. FLIM measurements of the solutions were made and the resulting gaussians were fit and extracted in FLIMFit. The gaussians were fit and the mean values were plotted on a $\log / \log$ scale against the corresponding solutions viscosity. The data was fit to the Förster Hoffman equation,

$$
\log \tau=\log \frac{z}{k_{0}}+x \log \eta
$$

965 Where $\tau$ is the lifetime in $\mathrm{ps}, \log \frac{z}{k_{0}}$ is treated as a variable, $\mathrm{x}$ is the slope, and $\eta$ is the viscosity in 966 centipoise. Fitting the data gave an equation of the form $y=0.09 x+7.7$. 
Figure 2-figure supplement 3.

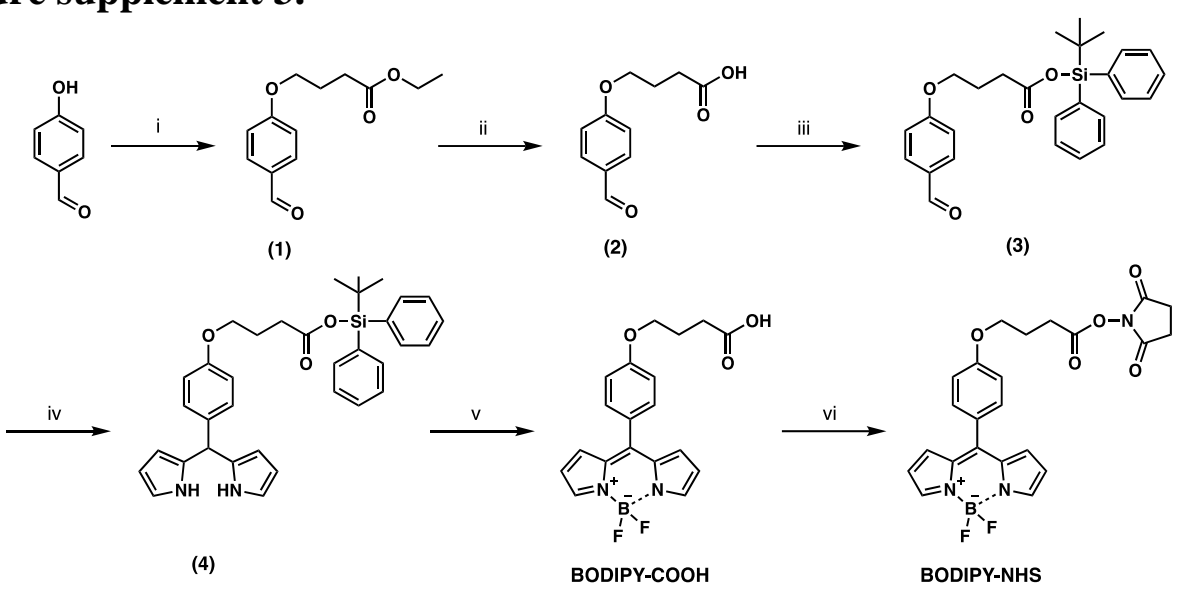

(4)

BODIPY-COOH

BODIPY-NHS

Figure 2-figure supplement 3. Synthesis scheme of BODIPY-NHS: (i) ethyl 4-bromobutyrate, $\mathrm{K}_{2} \mathrm{CO}_{3}$, DMF, rt, 24 h, $98 \%$ yield; (ii) $2 \mathrm{~N} \mathrm{KOH}, \mathrm{MeOH}$, reflux, 2 h, $96 \%$ yield; (iii) DMAP, $\mathrm{Et}_{3} \mathrm{~N}$, THF then tert-butyl(chloro)diphenylsilane, $0 \mathrm{oC}$ to rt, $20 \mathrm{~h}, 68 \%$ yield; (iv) pyrrole, TFA, rt, $1 \mathrm{~h}, 66 \%$ yield. (v) DDQ, $\mathrm{CH}_{2} \mathrm{Cl}_{2}, \mathrm{rt}, 1 \mathrm{~h}$ and then $\mathrm{BF}_{3} \cdot\left(\mathrm{OEt}_{2}\right), \mathrm{Et}_{3} \mathrm{~N}$, rt, overnight, $36 \%$ yield; (vi) N-hydroxysuccinimide, DCC, DMAP, THF, 0。C to rt, overnight, $64 \%$ yield. 
Figure 3

978
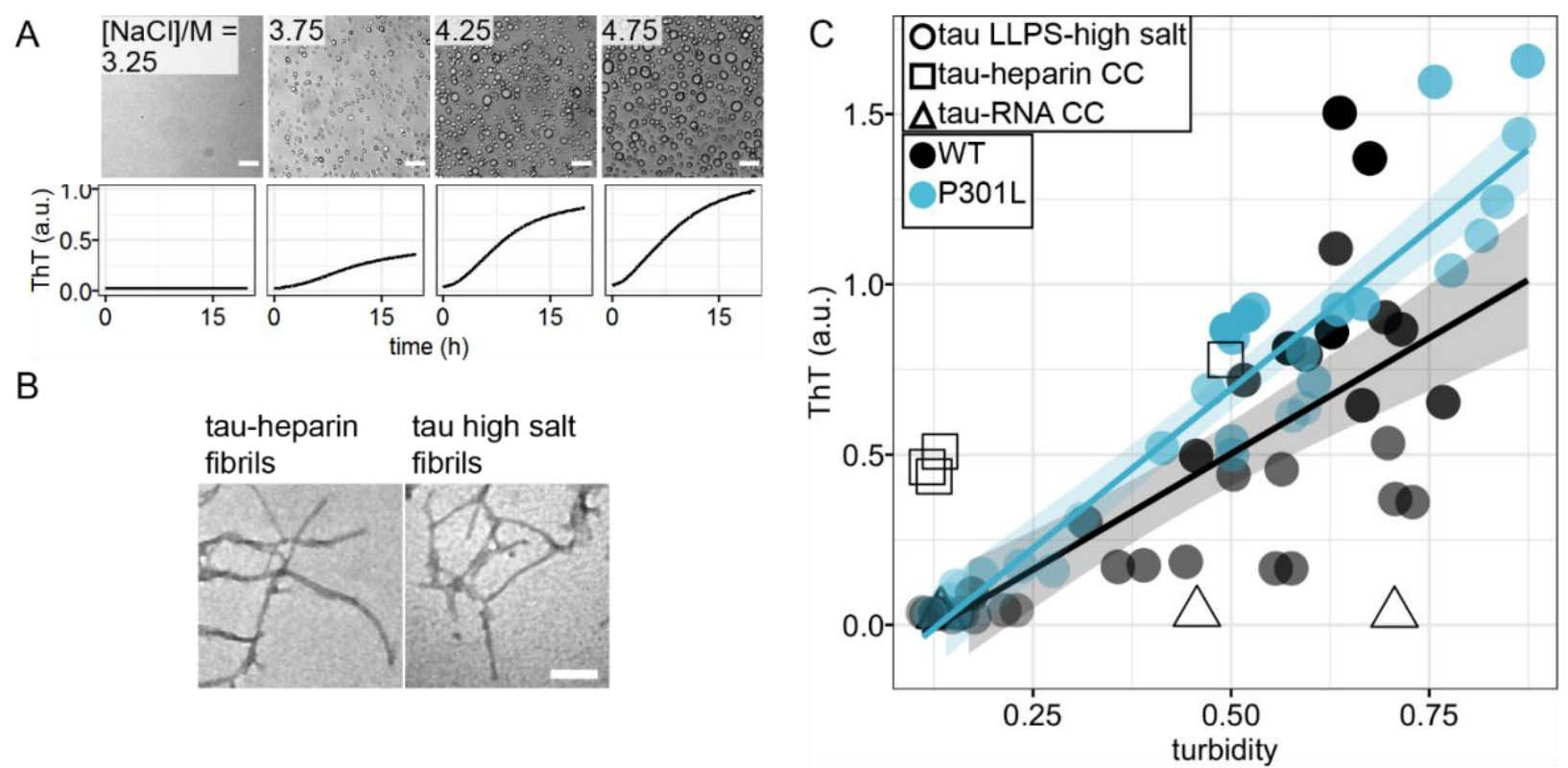

Figure 3. Correlation of LLPS-high salt and amyloid aggregation. A. Representative microscope images at $\mathrm{t}=0$ and ThT fluorescence at room temperature overnight of tau LLPS-high salt at various [ $\mathrm{NaCl}] .44 \mu \mathrm{M}$ tau187 was used. B. Representative TEM image of tau LLPS-high salt sample after overnight incubation, compared with tau-heparin fibrils. C. Correlation of initial turbidity and final ThT fluorescence of tau LLPS-high salt vs LLPS-CC. Solids line and shadow show linear regression and its standard error, respectively. $40 \mu M \pm 10 \%$ of tau187 was used. Samples come from 5 different batches of proteins. 
Figure 3-figure supplement 1. room temperature overnight of tau LLPS-high salt, tau-heparin LLPS-CC, tau-RNA
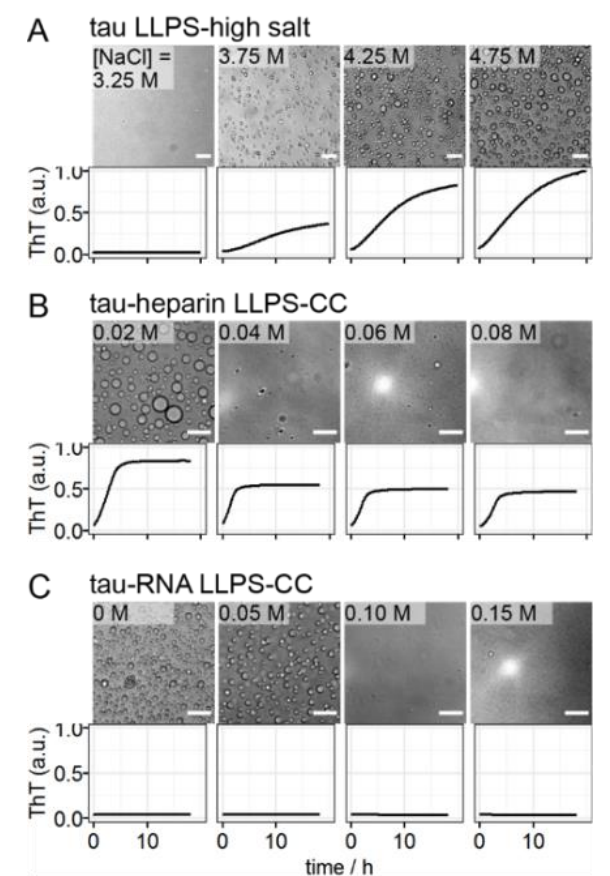

995 used. 
Figure 3-figure supplement 2.

997

998

1000

1001

1002

1003

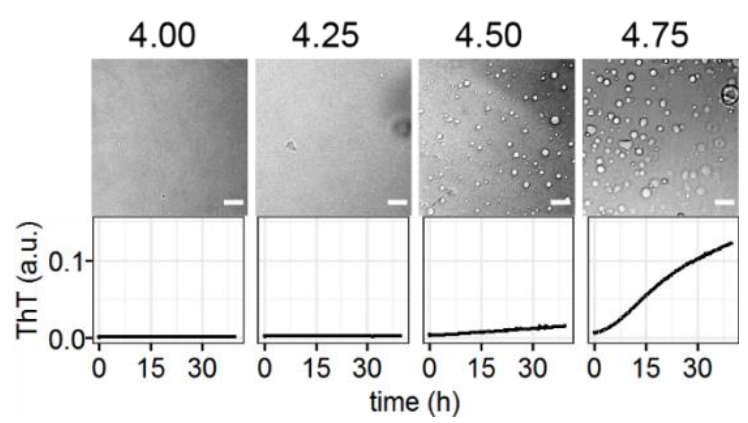

Figure 3-figure supplement 2. Correlation of LLPS-high salt and amyloid aggregation of 2N4R. $20 \mu \mathrm{M}$ 2N4R was incubated with varying [ $\mathrm{NaCl}]$. Microscope images were taken 10 minutes after mixing while ThT fluorescence readings were recorded overnight. Scale bar length was $25 \mu \mathrm{m}$. 
Figure 3-figure supplement 3.

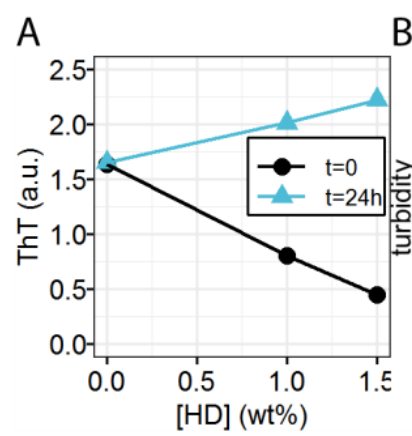

B LLPS-HS. A. Varying concentration of 1,6-hexanediol were added to fresh LLPS-HS sample $(\mathrm{t}=0)$ and matured LLPS-HS sample $(\mathrm{t}=24 \mathrm{~h})$. After hexanediol was added, samples were incubated for 24 hours and ThT fluorescence was recorded. B. Initial turbidity and final ThT fluorescence of LLPS-HS samples mixed with varying concentration of 1,6-hexanediol. In both $\mathrm{A}$ and $\mathrm{B}, 100 \mu \mathrm{M}$ tau187 and $3.0 \mathrm{M} \mathrm{NaCl}$ were used. 
Figure 4 LLPS high salt undergoes dehydration. A. Schematic diagram showing sensitive hydration shell in Electron Spin Echo Envelope Modulation (ESEEM) and Overhauser Nuclear Dynamic Polarization (ODNP). B. Representative 3 pulse-ESEEM of tau 187 at site 313 at solution (before) and upon addition of $3.75 \mathrm{M} \mathrm{NaCl}$ (after). 22.5\% Ficoll was used as glassing reagent. $\mathbf{C}$. Representative ODNP cross-relaxivity parameter $k_{\sigma}$ of tau187 at various sites at solution (before) and upon addition of $3.75 \mathrm{M} \mathrm{NaCl}$ (after). $250 \mu \mathrm{M}$ tau was used. For comparison, $k_{\sigma}$ of tau187 at site 322 upon tau-RNA complex coacervation was shown (322CC). 80\% reduction of $k_{\sigma}$ has been reported at site 313, 322 and 404 for tau-heparin fibrils (Pavlova, et al 2016), and is shown as dashed line. 
Figure 4-figure supplement 1.
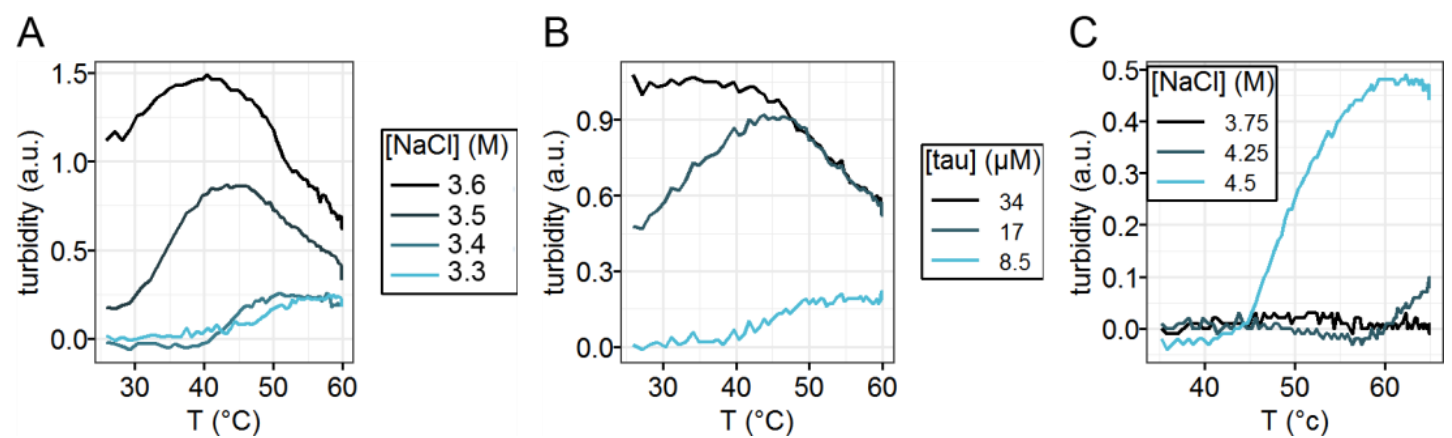

Figure 4-figure supplement 1. LCST behavior of tau LLPS-high salt. A-B. turbidity of tau187 LLPS-high salt upon heating at various [ $\mathrm{NaCl}$ ] with $34 \mu \mathrm{M}$ tau (A) and at varying [tau] with $3.9 \mathrm{M} \mathrm{NaCl}$ (B). C. Turbidity of 2N4R (C291SC322S) LLPS-high salt upon heating and cooling at various $[\mathrm{NaCl}] .20 \mu \mathrm{M}$ tau was used. In A-C, samples were prepared at room temperature $\left(25^{\circ} \mathrm{C}\right)$ and heated to $65^{\circ} \mathrm{C}$ at a rate of $\sim 1^{\circ} \mathrm{C} / \mathrm{min}$. 
Figure 4-figure supplement 2.

1040 


\section{Figure 4-figure supplement 3.}
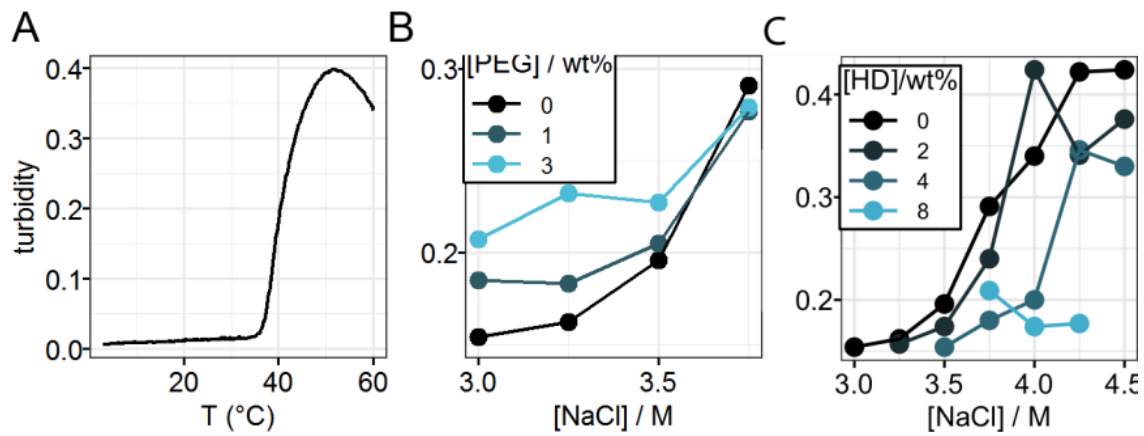

Figure 4-figure supplement 3. Hydrophobic interaction drives tau LLPS-high salt. A Turbidity of tau LLPS-high salt upon heating. $34 \mu \mathrm{M}$ tau187 and 3.5 M NaCl was used. turbidity vs $[\mathrm{NaCl}]$ of tau LLPS-high salt at varying [PEG]. C. Turbidity of tau LLPS-high salt at varying $[\mathrm{NaCl}]$ and $[\mathrm{HD}]$. In both $\mathrm{B}$ and $\mathrm{C}, 20 \mu \mathrm{M}$ tau187 was used. 
1055 Figure 4-source data 1.

1056

\begin{tabular}{ccc} 
site & \multicolumn{2}{c}{$\mathbf{k} / \mathbf{m M}$-1S-1 } \\
& before & after \\
\hline 303 & 0.031 & 0.009 \\
313 & 0.050 & 0.021 \\
322 & 0.048 & 0.023 \\
404 & 0.056 & 0.010 \\
4 OH-TEMPO & 0.092 & 0.097 \\
322CC & 0.050 & 0.045
\end{tabular}




\section{Figure 5}

A
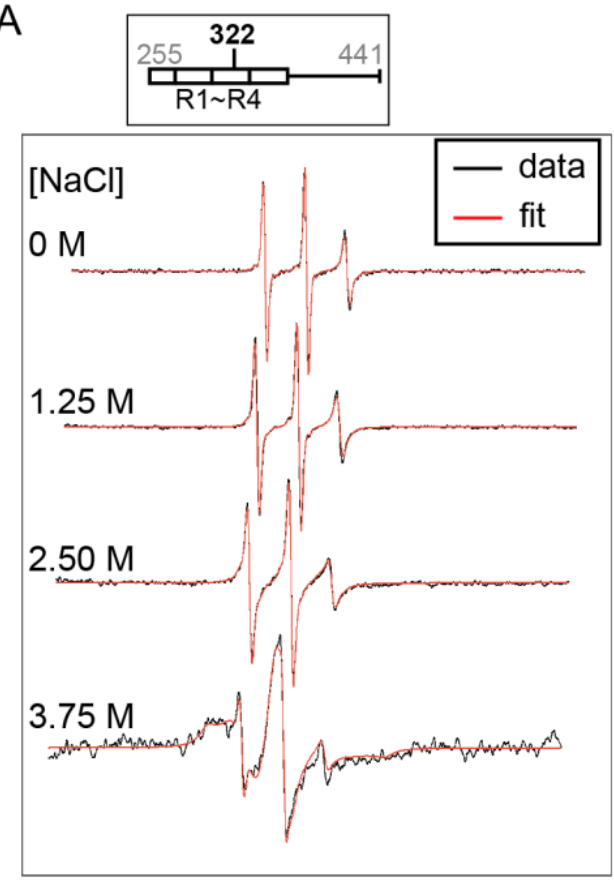

B

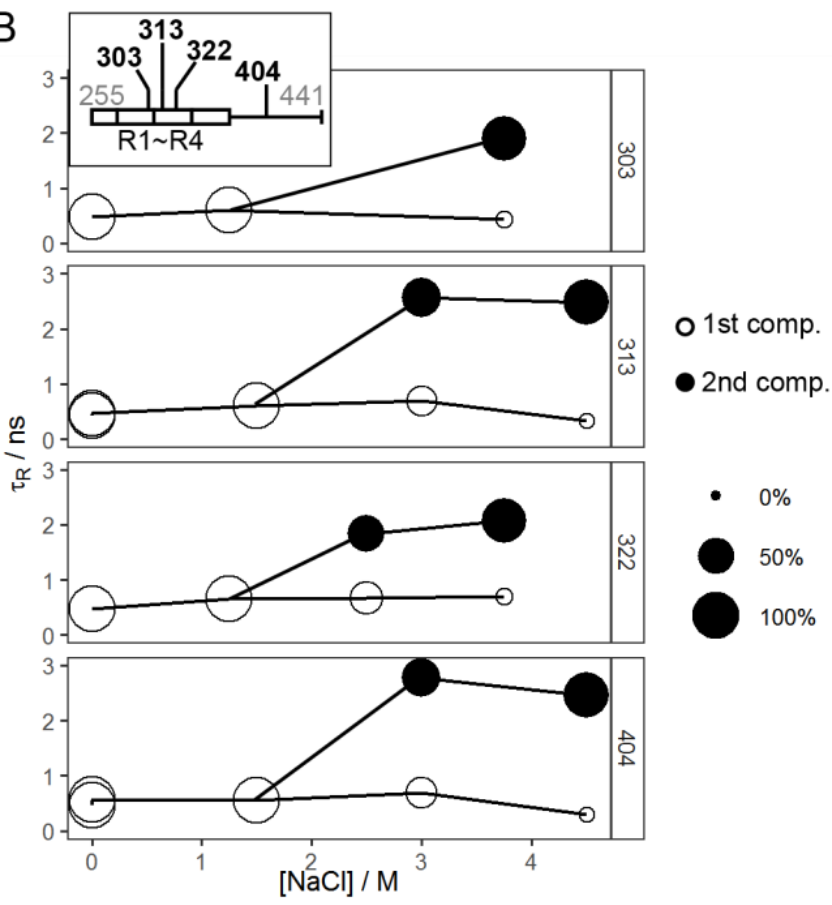

Figure 5. Site specific dynamics of tau187 upon LLPS-high salt. A. Representative X-band cwEPR lineshape of tau187 at site 322 at varying [ $\mathrm{NaCl}$. Black lines show the experiment data and red lines show the fit. Spectra are shifted to avoid overlap. B. Rotational correlation time, $\tau_{R}$, of tau187 at site 303, 313, 322 and 404 at varying [ $\mathrm{NaCl}$. Data at low [ $\mathrm{NaCl}]$ were fit with 1 component with the y-axis showing the $\tau_{R}$. Data at high $[\mathrm{NaCl}]$ were fitted with 2 components with the area of the disk proportional to the percentage of each component. 
Figure 5-figure supplement 1.

1072

1073
1074 1075 1076 1077 1078 1079 1080
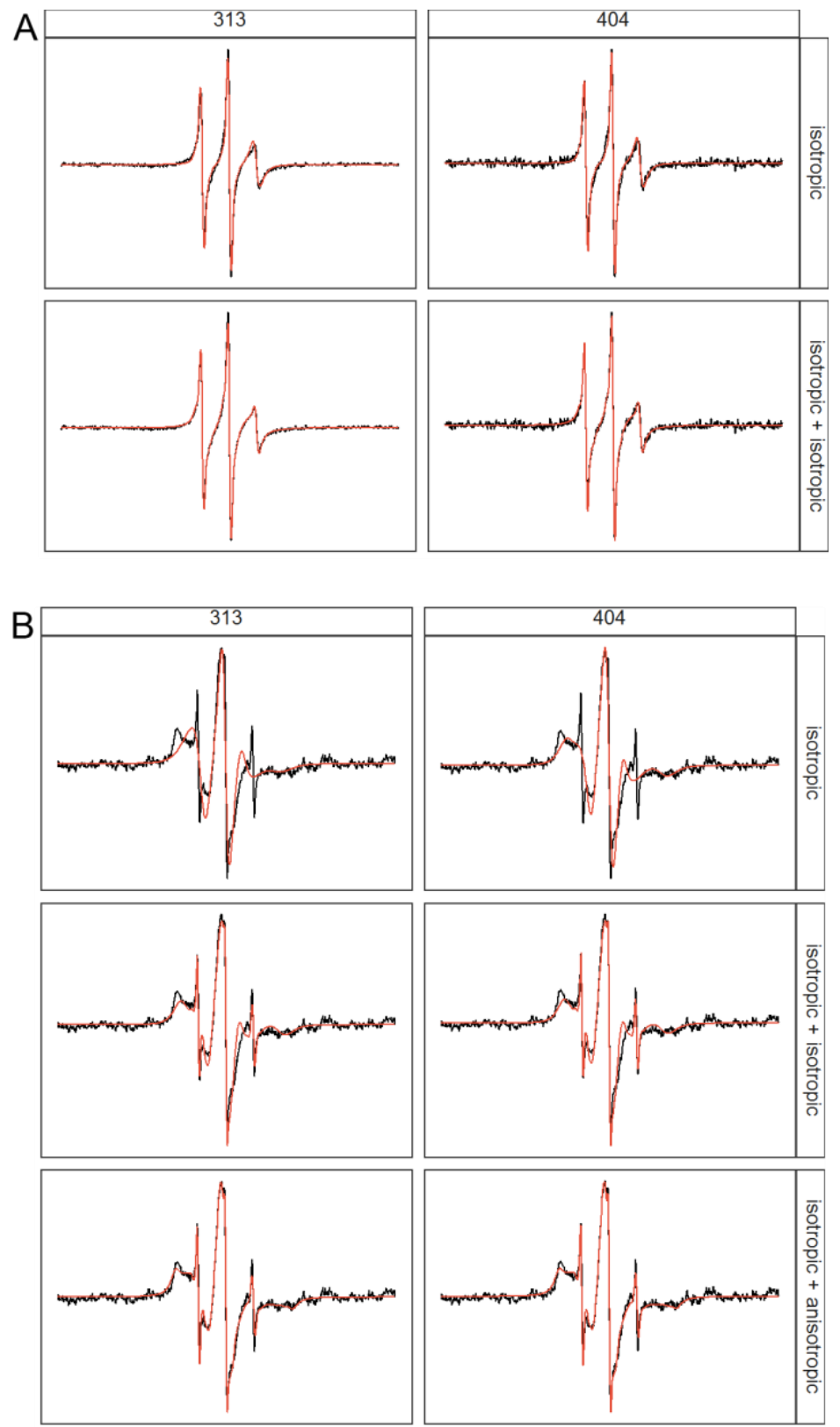

Figure 5-figure supplement 1. Multicomponent and Anisotropic fitting of cwEPR lineshapes. A. Comparison of 1 isotropic component fitting vs 2 isotropic component fitting of tau 187 at site 313 and 404 at intermediate $[\mathrm{NaCl}]$ of $3.0 \mathrm{M}$. B. Comparison of 1 isotropic, isotropic+isotropic and isotropic+anisotropic components fit of tau187 at site 313 and 404 at very high $[\mathrm{NaCl}]$ of $4.5 \mathrm{M}$. 
Figure 5-figure supplement 2.
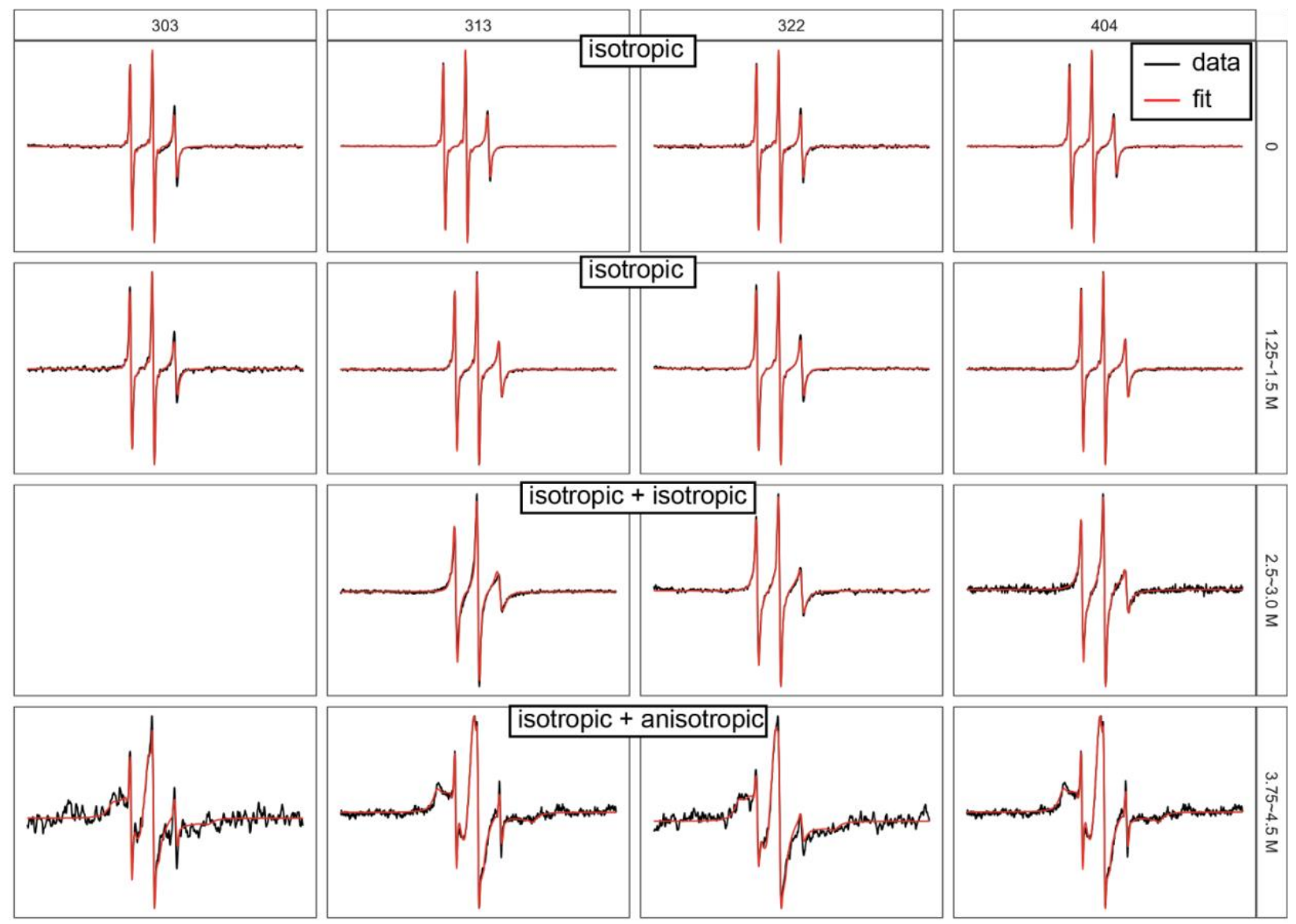

1084

1085

Figure 5-figure supplement 2. Best fit of cwEPR spectra of tau187 at varying sites and

1086

1087 varying [NaCl]. Experiment details and fit results were shown in Figure 5B. 


\section{Figure 5-figure supplement 3.}
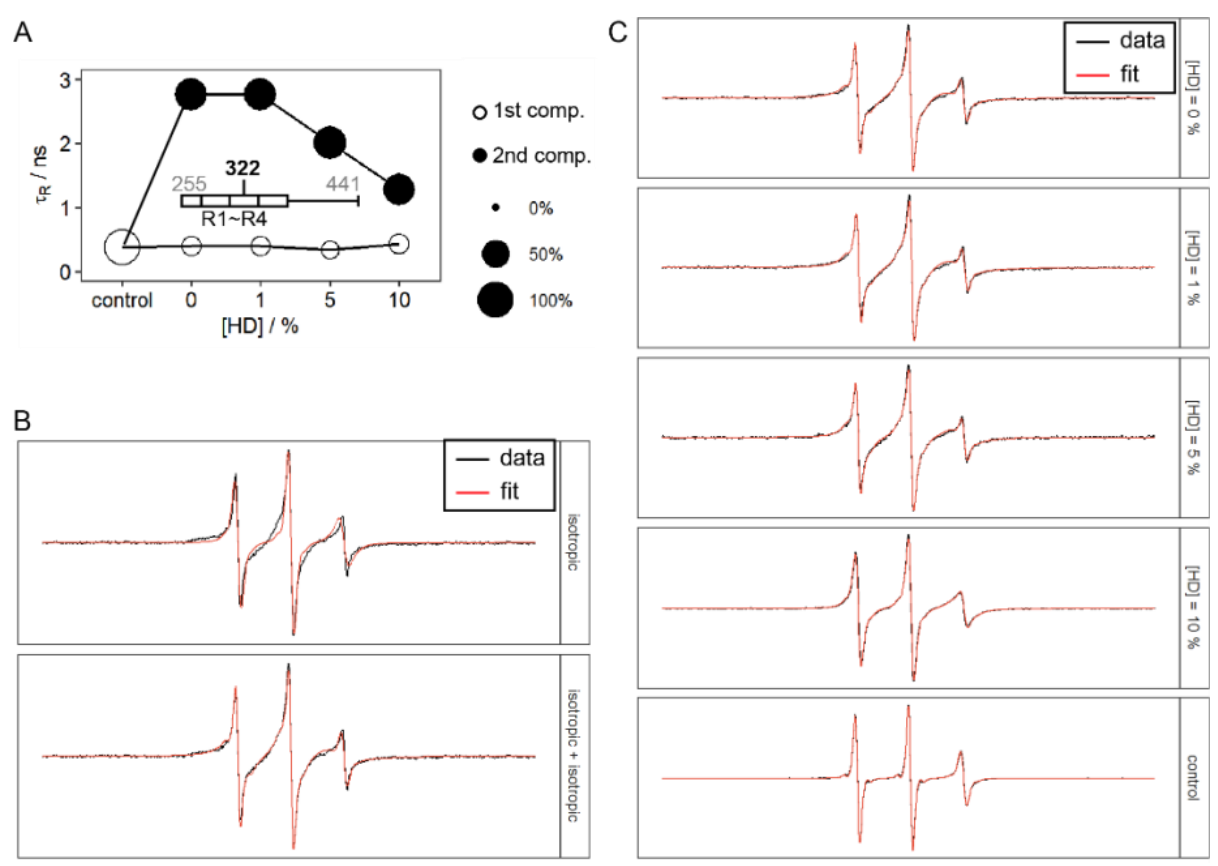

Figure 5-figure supplement 3. Effects of 1,6-hexanediol on tau LLPS-high salt. A. Rotational correlation time of tau LLPS-high salt at varying [HD]. $100 \mu \mathrm{M}$ tau187P301LC322SL and 2.5 M $\mathrm{NaCl}$ were used. B. Comparison of fitting cwEPR lineshape using one component vs two component. $[\mathrm{NaCl}]=2.5 \mathrm{M},[\mathrm{HD}]=0$. C. cwEPR lineshape and fitting results of data in A. 
Figure 5-figure supplement 4.

A
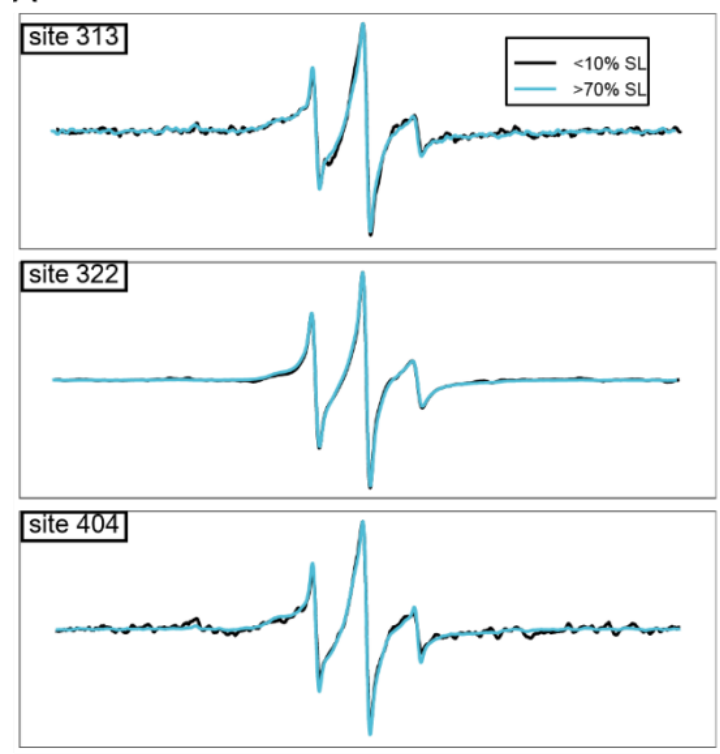

B

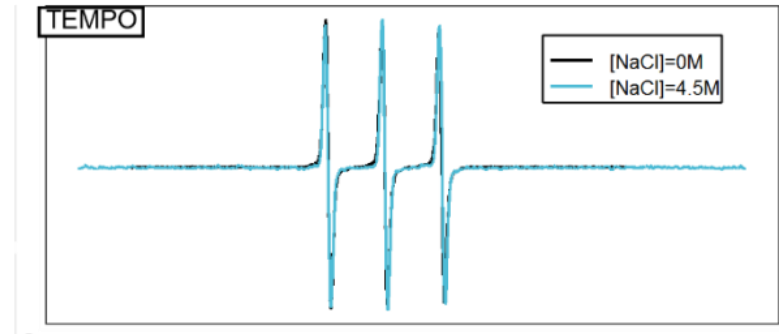

C

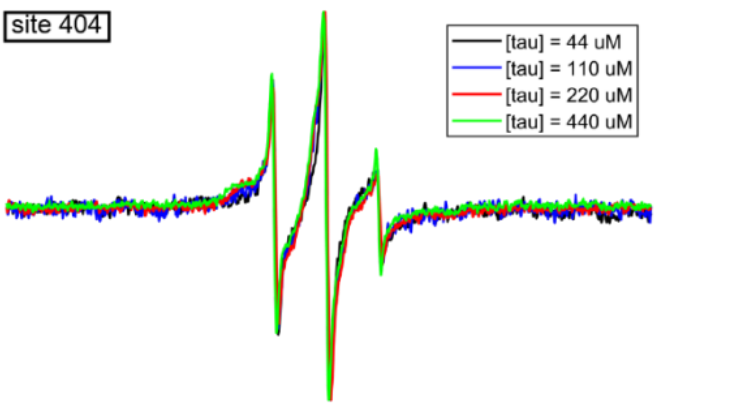

Figure 5-figure supplement 4. Spin dilution and concentration dependence of cwEPR

lineshape. In order to gain information on the oligomer structure, we investigated the sitespecific intermolecular interactions by cwEPR. Spin dilution, which consists in mixing 1 spinlabelled tau for 9 non-labeled tau, allows to diminish spin-spin dipolar coupling that could induce EPR lineshape broadening. By comparing cwEPR lineshape of spin-diluted sample vs non-spin-diluted sample, we can determine the population of spins that directly interact within a radius of $\sim 1.5 \mathrm{~nm}$. A. Effects of spin dilution on cwEPR lineshape of tau187 at site 313, 322 and 404. 3.0 M NaCl was used. B. Effects of 4.5 M NaCl on 4OH-TEMPO cwEPR lineshape. C. 


\section{Figure 5-source data 1.}

1113

$\begin{array}{cccccc}\text { site } & {[\mathbf{N a C l}] / \mathbf{M}} & \mathbf{t R}, \mathbf{1} / \mathbf{n s} & \mathbf{t R , 2} / \mathbf{n s} & \mathbf{p} \mathbf{1} / \mathbf{\%} & \mathbf{p} 2 / \% \\ 303 & 0.00 & 0.48 & & 100 & \\ & 1.25 & 0.61 & & 100 & \\ & 3.75 & 0.43 & 1.89 & 4 & 96 \\ 313 & 0.00 & 0.42 & & 100 & \\ & 1.50 & 0.61 & & 100 & \\ & 3.00 & 0.69 & 2.57 & 32 & 68 \\ 322 & 4.50 & 0.34 & 2.48 & 3 & 97 \\ & 0.00 & 0.47 & & 100 & \\ & 1.25 & 0.65 & & 100 & \\ & 2.50 & 0.68 & 1.83 & 40 & 60 \\ 404 & 3.75 & 0.70 & 2.08 & 4 & 96 \\ & 0.00 & 0.47 & & 100 & \\ & 1.50 & 0.56 & & 100 & \\ & 3.00 & 0.69 & 2.77 & 35 & 65\end{array}$

Figure 5-source data 1. Calculated parameters for cwEPR spectral simulation of tau187 mutants 10 minutes after LLPS at various [NaCl]. Experiment details and fit results were shown in Figure 5B. 

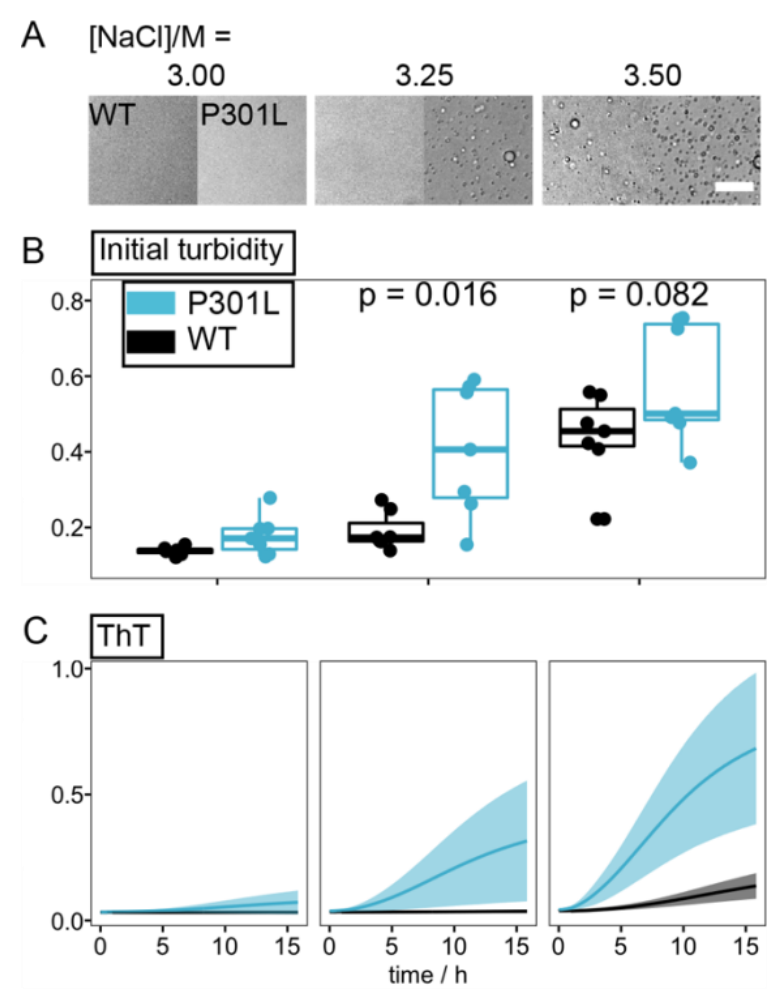

Figure 6 Effects of P301L mutation on LLPS and amyloid aggregation of tau at high salt mutants at varying $[\mathrm{NaCl}]$. B. Turbidity of corresponding samples in A. C. ThT fluorescence of samples in A after overnight incubation at room temperature. ThT fluorescence was scaled by a constant of $2 \times 10_{4}$ throughout the manuscript. Ribbons in $\mathrm{C}$ show standard deviation of readings from 5 different batches of proteins. 


\section{Figure 6-figure supplement 1.}

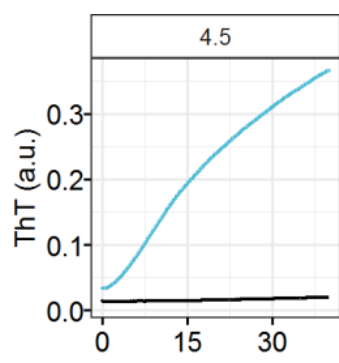
at high salt concentration. $20 \mu \mathrm{M} 2 \mathrm{~N} 4 \mathrm{R}$ was used. [NaCl] was shown in panel (unit: M). Samples were incubated at room temperature.

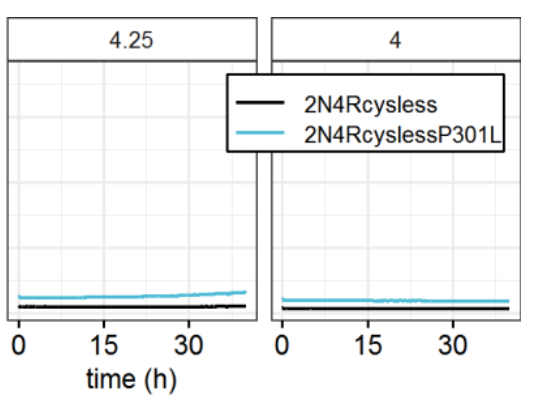


Figure 6-figure supplement 2.

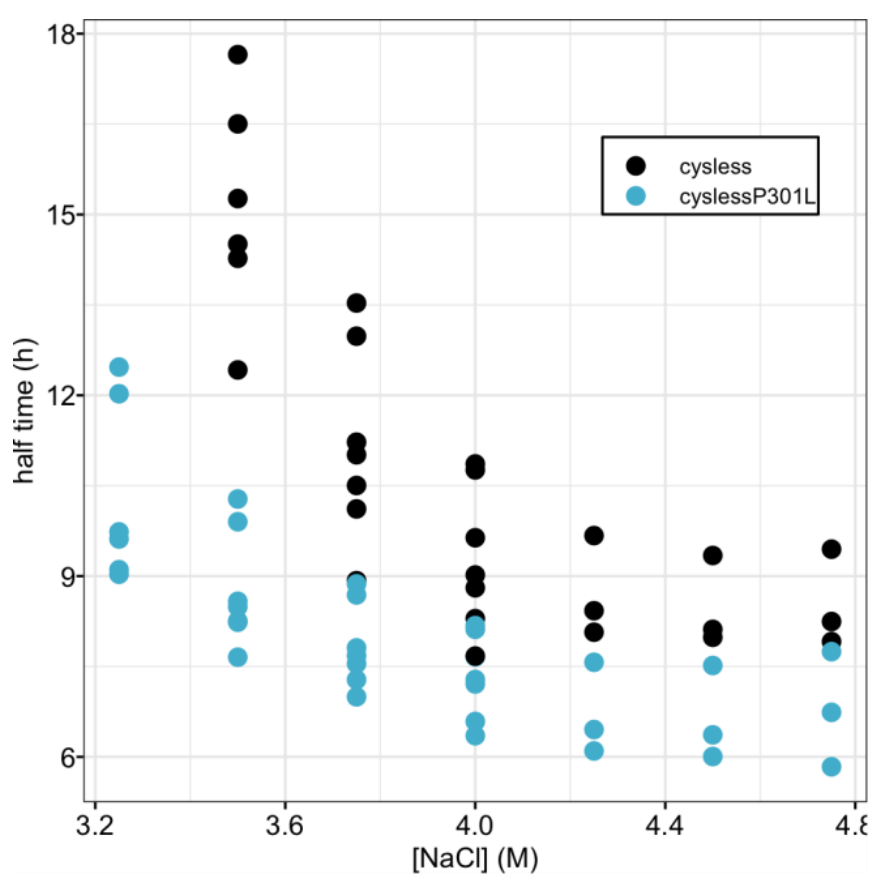

Figure 6-figure supplement 2. Effects of P301L mutation on half time of ThT fluorescence in high salt droplet conditions. ThT fluorescence readings were recorded at varying $[\mathrm{NaCl}]$ using $34 \mu \mathrm{M}$ tau187C291SC322S (cysless) and tau187C291SC322SP301L (cyslessP301L). Fluorescence readings were fit using a sigmoid function to extract the half time, defined as the time when ThT fluorescence reach half maximum. Data were obtained using $n>5$ biological independent repeats. 